\title{
BIMEROMORPHIC GEOMETRY OF RIGID ANALYTIC SURFACES
}

$\operatorname{AUTHOR}(\mathrm{S})$ :

MITSUI, KENTARO

CITATION:

MITSUI, KENTARO. BIMEROMORPHIC GEOMETRY OF RIGID ANALYTIC

SURFACES. International Journal of Mathematics 2011, 22(04): 483-513

ISSUE DATE:

2011-04

URL:

http://hdl.handle.net/2433/141851

RIGHT:

(C) 2011 World Scientific Publishing Co. 


\title{
BIMEROMORPHIC GEOMETRY OF RIGID ANALYTIC SURFACES
}

\author{
KENTARO MITSUI
}

\begin{abstract}
We show that any proper rigid analytic surface admits a relatively minimal regular model. Further, we give a criterion for relative minimality of proper regular rigid analytic surfaces.
\end{abstract}

\section{CONTENTS}

1. Introduction

2. Bimeromorphic Morphisms

3. Resolution of Singularities 6

4. Minimal Models 6

5. Minimal Models of Fibered Surfaces 7

6. Deformation of Divisors 9

7. Blowing-down of Exceptional Curves of The First Kind 15

8. Appendix 17

References $\quad 24$

\section{INTRODUCTION}

The rigid analytic GAGA theorems [18] connect rigid analytic geometry and algebraic geometry. One of their consequences is that classifying projective rigid analytic spaces is equivalent to classifying projective schemes over complete non-Archimedean valuation fields. However, similar to the complex analytic case, not all the proper rigid analytic spaces are derived from schemes. The situation naturally leads us to the classification problem for proper rigid analytic spaces. The first step toward the problem is to develop their bimeromorphic geometry. The present paper is focused on the two-dimensional case. In the following introduction, we call rigid analytic surfaces (resp. rigid analytic curves) surface (resp. curve) for short. Further, since we can always resolve their singularities (Theorem 3.1), we assume that they are regular.

The main results of the paper are the fundamental theorems on relatively minimal regular models of proper surfaces: the existence theorem (Theorem 4.2) and the criterion for relative minimality (Corollary 7.2). We also study the case where surfaces are fibered over quasi-compact curves. The paper consists of two parts. The first part is devoted to the study of bimeromorphic morphisms between surfaces and the second part contraction of divisors on surfaces. The main results follow from these studies.

In the first part, we show the factorization theorem (Theorem 2.2), which asserts that any bimeromorphic morphism between quasi-compact surfaces is a finite succession of blowing-downs. Although we can imitate the proof of the classical cases: the complex

2000 Mathematics Subject Classification. 14G22, 14E99. 
analytic case [12] and the scheme case [26], we also give another proof by a new technique that is interesting in its own right and useful in many occasions. For example, we use it for other studies of bimeromorphic morphisms and fibered surfaces. Our technique is based on the projectivity lemma (Lemma 2.4 ), which asserts that any proper morphism of relative dimension one is projective over a neighborhood of any point on the codomain. The lemma and the relative GAGA theorems [18] imply that the morphism is the analytification of a projective morphism between schemes whereas the similar lemma implies nothing in the complex analytic case for lack of relative GAGA theorems. After proving the lemma, the factorization theorem follows from the corresponding theorem on schemes [26].

Let us explain the proof of the lemma. Fix a point on the codomain and let $C$ be the fiber over the point. Since relative ampleness of line bundles is an open condition [9], we have only to construct a line bundle on a neighborhood of $C$ whose restriction to $C$ is ample. To this end, we prove that any proper rigid analytic space of dimension one is projective. Then we extend an ample divisor of $C$ to a neighborhood by the topological results [16] and [29]. The resulting divisor gives a desired line bundle. The projectivity lemma also plays key role in the second part.

In the second part, we show the contraction theorem (Theorem 7.1), which asserts that any exceptional curve of the first kind on any surface can be contracted to a regular point. In the complex analytic case, the similar theorem can be shown in the following way [17]. Choose two distinct points $P$ and $Q$ on the exceptional curve $D$. Take two disjoint smooth prime divisors $E$ and $F$ on a small neighborhood $U$ of $D$ such that $D \cap E=P$ and $D \cap F=Q$. Assume that each of the intersection multiplicities is equal to one. Replace $U$ with a smaller one, we can contract the divisor $E$ to a smooth point. Let $V$ be the resulting space and $G$ be the image of $D$. Then the deformation theory of smooth divisors gives a defining function $g$ of $G$ on $V$. Let $f_{P}$ be the pull-back of $g$ on $U$, which is a defining function of $D+E$. Repeat the same procedure for $Q$ to obtain a defining function $f_{Q}$ of $D+F$. Then the two analytic functions $f_{P}$ and $f_{Q}$ define the morphism $\phi$ from $U$ to a two-dimensional polydisk, which gives a desired contraction.

In the rigid analytic case, Ueno gives the contraction theorem [28] in a particular case by the above method. However, his proof works only when the base field is algebraically closed. The following two difficulties arise when we adapt the method for the general case where the base field is general and the surface is not necessarily smooth but regular. The first difficulty is that in general we can not contract $E$ to obtain the appropriate divisor $G$ and space $V$. Even if possible, we run into the second difficulty, that in general the morphism $\phi$ does not give a desired contraction since it is not necessarily an isomorphism on the complement of $D$.

The first difficulty is resolved by the deformation theory of singular divisors. First, we develop the deformation theory by non-Archimedean analysis. Next, we apply the theory to obtain the morphism $\phi$ in the following way. Take a prime divisor $E^{\prime}$ that intersects $D$ only at the point $P$ with multiplicity one. Since the obstruction to the deformation of the singular divisor $D+E^{\prime}$ vanishes, we obtain a defining function $f_{P}$ of $D+E^{\prime}$. Repeat the same procedure for $Q$ to obtain $f_{Q}$. Then the two analytic functions $f_{P}$ and $f_{Q}$ define the morphism $\phi$ form $U$ to a two-dimensional polydisk.

The second difficulty is overcome by the projectivity lemma in the first part. Since the properness criterion (Proposition 7.3, cf. [3, I.(8.2)]) shows that the morphism $\phi$ is proper over a neighborhood of the origin of the polydisk, the lemma is applicable to $\phi$. Therefore, the lemma and the relative GAGA theorems imply that there exists a neighborhood of $D$ 
that is the analytification of a scheme. Then the contraction theorem follows from the corresponding theorem on schemes [26].

In the appendix, since we have no appropriate reference, we prove that any proper rigid analytic space of dimension one is projective, the theorem which is used in the proof of the projectivity lemma. To prove it, we review and develop the fundamental theory of finite morphisms, cohomology groups of abelian sheaves, and divisors. The rest of the appendix is devoted to the foundation of the intersection theory on proper surfaces in order to characterize exceptional curves by their self-intersection numbers. Further, we prove the Riemann-Roch theorem for proper smooth surfaces (Theorem 8.23).

Notations and Conventions. We fix a complete non-Archimedean valuation field $K$ with a non-trivial valuation and assume that rigid analytic spaces are defined over $K$. We mainly use the terminologies and notations of [5]. However, we make a modification of the definition of smoothness. Let $X$ be a rigid analytic space. When the local ring $\mathscr{O}_{X, x}$ is regular for all $x \in X$, the rigid analytic space $X$ is said to be regular. When the base change $X \times_{K} K^{\prime}$ for any extension $K^{\prime} / K$ of complete valuation fields is regular, the rigid analytic space $X$ is said to be smooth (or geometrically regular). If the base field $K$ is perfect, then regularity is equivalent to smoothness (see [14]).

The dimension of a rigid analytic space $X$ is the supremum of the Krull dimension of the local ring $\mathscr{O}_{X, x}$ for all $x \in X$. When the Krull dimension is constant for all $x \in X$, the rigid analytic space $X$ is said to be of pure dimension. A curve (resp. surface) is a reduced separated rigid analytic space of pure dimension one (resp. two).

By $K(x)$ let us denote the residue field at a point $x$ on a rigid analytic space, which is a finite extension of $K$. We set a complete valuation on $K(x)$ which is the unique extension of that on $K$. By $C(D)$ let us denote the field of constant functions $H^{0}\left(D, \mathscr{O}_{D}\right)$ on a proper rigid analytic space $D$, which is a finite extension of $K$.

An element of the set

$$
\left\{|a|^{b} \in \mathbb{R} \mid a \in K^{\times}, b \in \mathbb{Q}\right\}
$$

is called a special real number.

Terminology in the divisor theory and intersection theory for rigid analytic spaces is explained in the appendix.

\section{BIMEROMORPHIC MORPHISMS}

2.1. Blowing-ups and Blowing-downs. A proper surjective morphism $\phi: X \rightarrow Y$ of rigid analytic spaces is called a bimeromorphic morphism if there exist nowhere dense analytic subsets $S$ and $T$ of $X$ and $Y$ respectively such that the restriction $\phi \mid X-S \rightarrow Y-T$ is an isomorphism. Note that Corollary 7 in [5, 9.1.4] implies that the subsets $X-S$ and $Y-T$ are admissible open subsets.

Let $\phi: X \rightarrow Y$ be a bimeromorphic morphism between quasi-compact surfaces with the analytic subsets $S$ and $T$ in the above definition. Then the analytic subset $S$ is the union of a finite number of points and proper curves. By Proposition 4.6 in [4], the fiber $\phi^{-1}(y)$ is a point or a proper connected curve for each $y \in Y$. By the same proposition, we may assume that the analytic subset $T$ is a finite number of points and that the analytic subset $S$ is equal to the preimage $\phi^{-1}(T)$. An element $y$ of $T$ is called a fundamental point for $\phi$ and the curve $\phi^{-1}(y)$ an exceptional curve for $\phi$.

Conrad defined and studied blowing-ups of rigid analytic spaces in $[9,4.1]$ (see also [24] for algebraically closed base field cases). The blowing-ups provide examples of bimeromorphic morphisms since the blowing-ups are proper (Corollary 2.3.9 in [9]). Any blowing-up of an affinoid space is isomorphic to the analytification of the algebraic one. 
We only treat regular surfaces and their blowing-ups at a point. Let $\pi: \widetilde{X} \rightarrow X$ be the blowing-up of a regular surface $X$ at a point $p$. Then the resulting surface is also regular. In this case, we say that the morphism $\pi$ is the blowing-down of $\widetilde{X}$ along the divisor $\pi^{-1}(p)$. The following extension theorem shows the uniqueness of such contraction of divisors.

Theorem 2.1 (extension theorem). Let $T$ be a nowhere dense analytic subset of a normal rigid analytic space $Y$. Assume that a morphism $f: Y-T \rightarrow Z$ of rigid analytic spaces satisfies the following condition: There exists an admissible covering $\left\{U_{i}\right\}_{i \in I}$ of $Y$ such that each image $f\left(U_{i}-T\right)$ is contained in an admissible affinoid open subset of $Z$. Then there exists a unique morphism $g: Y \rightarrow Z$ such that the restriction $g \mid Y-T \rightarrow Z$ is equal to $f$.

Proof. First, we assume that the rigid analytic spaces $Y$ and $Z$ are affinoid spaces $\operatorname{Sp} A$ and $\mathrm{Sp} B$ respectively. Applying the Riemann extension theorem ([2, §3]) to the pull-backs of the analytic functions on $\operatorname{Sp} B$ under $f$, we obtain the unique $K$-algebra homomorphism $B \rightarrow A$. The uniqueness follows from the uniqueness in the Riemann extension theorem. Therefore, we obtain the desired morphism $g: \operatorname{Sp} A \rightarrow \operatorname{Sp} B$. This construction is compatible with the restriction of $\operatorname{Sp} A$ to any admissible affinoid open subset. Thus, by assumption, the general case follows by a gluing argument (Proposition 1 in [5, 9.3.3]).

In the following subsections, we give two proofs of the following theorem.

Theorem 2.2 (Hopf's theorem). Any bimeromorphic morphism between quasi-compact regular surfaces is a finite succession of blowing-downs.

2.2. The First Proof. In this subsection, we prove Hopf's theorem by the above extension theorem.

We prove Hopf's theorem by induction on the number of the irreducible components of the union of all the exceptional curves. Let $y$ be one of the fundamental points for a bimeromorphic morphism $\phi: X \rightarrow Y$. We take an admissible affinoid open subset $V$ and two analytic functions $f^{\prime}$ and $g^{\prime}$ on $V$ that define the point $y$ and generate the maximal ideal of the local ring $\mathscr{O}_{Y, y}$. We may assume that the subset $V$ does not contain the other fundamental points. Put $U:=\phi^{-1}(V)$. Since the morphism $\phi$ is quasi-compact, the admissible open subset $U$ is quasi-compact. Put $f:=\phi^{*} f^{\prime}$ and $g:=\phi^{*} g^{\prime}$.

Claim. The principal divisors $(f)$ and $(g)$ are given by $C+E$ and $D+F$ respectively where the divisors $C, D, E$, and $F$ satisfy the following conditions.

(1) The divisors $C$ and $D$ are prime divisors that are not contained in $\phi^{-1}(y)$.

(2) The equalities $\operatorname{Supp} E=\operatorname{Supp} F=\phi^{-1}(y)$ hold.

Proof. Since the restriction $\phi \mid U-\phi^{-1}(y) \rightarrow V-\{y\}$ is an isomorphism, the claim follows from Corollary 2.2.9 in [7].

Claim. Replacing suitable analytic functions $f^{\prime}$ and $g^{\prime}$, we may assume $E=F$ in the above claim.

Proof. Since the admissible open subset $U$ is quasi-compact, by Theorem 8.9, we have a finite locally principal covering of $U$ for all prime divisors that appear in the finite sum $(f)$ or $(g)$. Then the above claim follows from the following lemma.

Lemma 2.3. Let I be an ideal of an affinoid algebra A. Let $f$ and $g$ be two elements of $A$. Assume $f \notin I$ or $g \notin I$. Then there exists a positive real number $\varepsilon$ satisfying the following condition. For all two non-zero elements $\alpha$ and $\beta$ of $K$ such that $|\alpha|<\varepsilon$ and $|\alpha|<\varepsilon$, neither $f+\alpha g$ nor $\beta f+g$ is contained in $I$. 
Proof. Let $\psi: B(1)^{2} \rightarrow A^{2}$ be the continuous map defined by $\psi(s, t)=(f+s g, t f+g)$. Assume $f \in I$ or $g \in I$. Without loss of generality, we may assume $f \in I$. Then the condition is fulfilled on an open neighborhood of $(0,0)$ minus $\{s=0\}$. Therefore, there exists a desired positive real number $\varepsilon$ in this case. Assume $f \notin I$ and $g \notin I$. By Proposition 2 in [5, 3.7.2], the subset $I \times A \cup A \times I$ of $A^{2}$ is closed. Therefore, the complement of $\psi^{-1}(I \times A \cup A \times I)$ is an open neighborhood of $(0,0)$. This proves the second case.

Claim. The ratio $(f: g)$ defines a morphism from $U$ to $\mathbb{P}_{V}^{1}$ over $V$.

Proof. We have only to show that the two divisors $C$ and $D$ do not intersect. Suppose that these two divisors intersected. Let $p$ be one of the intersections. Then the point $p$ is contained in $\phi^{-1}(y)$.

Let $\pi: D^{n} \rightarrow D$ be the normalization of $D$. The restriction $\phi \circ \pi \mid D^{n}-(\phi \circ \pi)^{-1}(y) \rightarrow$ $\left\{g^{\prime}=0\right\}-\{y\}$ is an isomorphism. Since $D^{n}$ is separated, there exists a finite family $\left\{W_{q}\right\}_{q \in \pi^{-1}(p)}$ of disjoint admissible affinoid open subsets of $D^{n}$ such that each element $W_{q}$ contains $q$. Put $W:=\bigcup_{q \in \pi^{-1}(p)} W_{q}$ and $h:=\pi^{*} f$. Taking an admissible affinoid covering of $D^{n}$ and applying Lemma 2.3 in [16], we obtain a special real number $\varepsilon$ such that $\left\{z \in D^{n}|| h \mid \leq \varepsilon\right\} \subset W$. Put $G:=\left\{x \in X|| f^{\prime} \mid \leq \varepsilon, g^{\prime}=0\right\}$. Then the preimage of $G$ under $\phi$ is an admissible affinoid open subset of $D^{n}$. Thus, Theorem 2.1 shows that the rigid space $G$ is isomorphic to an admissible affinoid open subset of $D^{n}$. Therefore, the pull-back $h$ is a parameter at the point $\pi^{-1}(p)$ while the analytic function $\pi^{*} g$ vanishes.

We may make the same argument with respect to $C$. The two results show that the two analytic functions $f$ and $g$ generate the maximal ideal of the local ring $\mathscr{O}_{X, p}$ This contradicts the assumption that the point $y$ is a fundamental point for $\phi$.

Let $\pi: \widetilde{Y} \rightarrow Y$ be the blowing-up of $Y$ at the point $y$. The above claim enables us to define the morphism $\widetilde{\phi}: X \rightarrow \widetilde{Y}$ of rigid analytic spaces such that $\phi=\pi \circ \widetilde{\phi}$. Then the number of the irreducible components of the union of all the exceptional curves for $\widetilde{\phi}$ is less than that for $\phi$. Therefore, Hopf's theorem follows by induction on this number.

2.3. The Second Proof. In this subsection, we prove Hopf's theorem by algebraization. To algebraize bimeromorphic morphisms locally, we prepare the following lemma.

Lemma 2.4. Let $\pi: X \rightarrow Y$ be a proper morphism of rigid analytic spaces. Assume that the fiber at a point $p$ on $Y$ is of dimension at most one. Then there exists an admissible open subset $U$ of $Y$ containing $p$ such that the base change $\pi \times_{Y} U: X \times_{Y} U \rightarrow U$ is projective.

Proof. Put $X_{p}:=\pi^{-1}(p)$. By Theorem 8.12 , the fiber $X_{p}$ is a projective rigid analytic space over $K(p)$. By Chow's theorem (Theorem 8.3) the rigid analytic space $X_{p}$ is the analytification of a projective scheme over $K(p)$. By Proposition 7.1.32 in [21], we have an effective ample Cartier divisor $\sum_{i \in I} a_{i} D_{i}$ on $X_{p}$.

We fix $i \in I$. Since the morphism $\pi$ is proper, there exists two admissible affinoid open subsets $P$ and $Q$ of the preimage of an admissible affinoid open subset $V$ of $Y$ under $\pi$ such that $D_{i} \in P$ and $P \Subset_{V} Q$. Since $Q \not \subset X_{p}$, there exists an effective Cartier divisor $E$ on $X_{p}$ such that $\operatorname{Supp} E \not \subset Q$. Then, by Corollary 7.3.23 in [21], there exist a meromorphic function $f$ on $X_{p}$ and a positive integer $b$ such that the inequality $(f) \geq D_{i}-b E$ holds.

We may assume $P=\left\{x \in Q|| g_{1}(x)|\leq \varepsilon, \ldots,| g_{n}(x) \mid \leq \varepsilon\right\}$ where the family $\left\{g_{1}, \ldots, g_{n}\right\}$ is an affinoid generating family of $Q$ over $V$ and $\varepsilon$ is a special real number less than one. Put $R:=\bigcup_{j=1, \ldots, n} Q\left\langle g_{1} / \varepsilon, \ldots, g_{n} / \varepsilon, \varepsilon / g_{j}\right\rangle$. Replacing $\varepsilon$ with a larger special real number, we may assume $X_{p} \cap R \cap \operatorname{Supp}(f)=\emptyset$. 
We choose an analytic function $h$ on $Q$ whose restriction to the analytic subset $Q \cap X_{p}$ is equal to the restriction of $f$ to it. Then the relations $X_{p} \cap R \subset R-\{h=0\} \subset R$ hold. Applying Lemma 2.3 in [16], we obtain a connected admissible affinoid open subset $W_{i}$ of $V$ containing $p$ such that $\{h=0\} \cap \pi^{-1}\left(W_{i}\right) \subset(Q-R) \cap \pi^{-1}\left(W_{i}\right)$. Then we obtain the Cartier divisor $\pi^{-1}\left(W_{i}\right) \cap P \cap\{h=0\}$ on $Q$. Thus, by Lemma 1.1 in [29], we obtain the Cartier divisor $F_{i}$ on $\pi^{-1}\left(W_{i}\right)$ such that $\left.F_{i}\right|_{X_{p}} \geq D_{i}$.

Repeating the same procedure for each $i \in I$, we obtain the intersection $W^{\prime}$ of the admissible open subsets $W_{i}$ and the sum $F^{\prime}$ of the Cartier divisors $\left.F_{i}\right|_{\pi^{-1}\left(W^{\prime}\right)}$ on $\pi^{-1}\left(W^{\prime}\right)$. By Proposition 7.5.5 in [21], the Cartier divisor $\left.F^{\prime}\right|_{X_{p}}$ is ample. Applying Theorem 3.2.9 in [9] to the line bundle defined by the Cartier divisor $F^{\prime}$, we conclude the proof of the lemma.

Proof of Hopf's theorem. Let $\phi: X \rightarrow Y$ be a bimeromorphic morphism between quasicompact regular rigid analytic spaces of pure dimension two. Since the statement is local with respect to $Y$, we may assume that the morphism $\phi$ has a single fundamental point $y$.

By the above lemma and Chow's theorem (Theorem 8.3), we may algebraize the bimeromorphic morphism $\phi$ over an admissible affinoid open subset containing $y$. Therefore, the theorem follows from Hopf's theorem for two-dimensional Noetherian regular schemes ([26, Chapter 4, p.55]).

\section{Resolution of Singularities}

Schoutens gives the resolution of singularities of rigid analytic spaces over an arbitrary algebraically closed base field of characteristic zero in [25]. Here, we give the resolution of singularities of rigid analytic spaces of dimension two over an arbitrary base field.

Theorem 3.1. For any quasi-compact reduced rigid analytic space $X$ of dimension two, there exists a quasi-compact regular rigid analytic space $Y$ and a bimeromorphic morphism $Y \rightarrow X$.

Proof. First, note that any affinoid algebra is excellent $([7,1])$. Therefore, the regular locus of any reduced affinoid space is a non-empty Zariski open subset. We normalize $X$ (see [7, 2.1]) and blow up the resulting rigid analytic space alone the singular locus (see Definition 4.1.1 in [9]). We have only to show that we can obtain a regular rigid analytic space in a finite succession of this procedure. To show this, we may assume that $X$ is an affinoid space. Then since the analytic normalization and blowing-up of an affinoid space coincide with the analytification of the algebraic ones, the termination follows from the remark $\mathrm{B}$ in the introduction of [20].

\section{Minimal Models}

A relatively minimal regular surface (resp. relatively minimal smooth surface) is a proper regular surface $X$ (resp. proper smooth surface) such that any bimeromorphic morphism from $X$ to a proper regular surface (resp. proper smooth surface) is an isomorphism. A relatively minimal regular model (resp. relatively minimal smooth model) of a proper regular surface $X$ (resp. proper smooth surface) is a relatively minimal regular surface $Y$ (resp. relatively minimal smooth surface) such that there exists a bimeromorphic morphism from $X$ to $Y$.

To show the existence of relatively minimal regular models and relatively minimal models, we prove the following proposition. 
Proposition 4.1. Let $\pi: \widetilde{X} \rightarrow X$ be the blowing-up of a proper regular surface $X$ at a point p. Then the inequality

$$
\operatorname{dim}_{K} H^{1}\left(\widetilde{X}, \Omega_{\widetilde{X}}^{1}\right)>\operatorname{dim}_{K} H^{1}\left(X, \Omega_{X}^{1}\right)
$$

holds. For any line bundle $\mathscr{L}$ on $X$, the equality

$$
\operatorname{dim}_{K} H^{q}\left(\widetilde{X}, \pi^{*} \mathscr{L}\right)=\operatorname{dim}_{K} H^{q}(X, \mathscr{L})
$$

holds for all $q$.

Proof. We take an admissible affinoid open subset $U$ of $Y$ such that two analytic functions on $U$ define the point $p$ and generate the maximal ideal of the local ring $\mathscr{O}_{Y, p}$. Then the assertion follows from the Mayer-Vietoris sequence (Proposition 8.6) and the local calculation in [26, Chapter 5, pp.59-65].

Proposition 4.1 and Hopf's theorem (Theorem 2.2) show the existence of relatively minimal regular models and relatively minimal smooth models.

Theorem 4.2 (existence of relatively minimal regular models). Any proper regular surface admits a relatively minimal regular model.

Theorem 4.3 (existence of relatively minimal smooth models). Any proper smooth surface admits a relatively minimal smooth model.

\section{Minimal Models of Fibered Surfaces}

A (regular) fibered surface with fibers of arithmetic genus $g$ (over a regular curve) is a triple $(X, S, \pi)$ where $X$ is a regular surface, $S$ is a regular curve, and $\pi$ is a proper flat morphism from $X$ to $S$ satisfying the following condition. There exists a nowhere dense analytic subset $S_{0}$ of $S$ such that for all $p \in S-S_{0}$, the fiber $\pi^{-1}(p)$ is an irreducible curve of arithmetic genus $g$ over $K(p)$.

Remark. The flatness of the morphism $\pi$ is equivalent to the surjectivity of the morphism $\pi$.

We define algebraic fibered surfaces over algebraic regular curves in the same way. An (algebraic) fibered surface $(X, S, \pi)$ is said to be smoothly fibered if the projection $\pi$ is smooth. An (algebraic) fibered surface $(X, S, \pi)$ is said to be proper if the total space $X$ and the base space $S$ are proper.

A relatively minimal fibered surface is a fibered surface $(X, S, \pi)$ satisfying the following condition. For any fibered surface $(Y, S, \rho)$, any bimeromorphic morphism from $X$ to $Y$ over $S$ is an isomorphism. A relatively minimal model of a fibered surface $(X, S, \pi)$ is a relatively minimal fibered surface $(Y, \rho, S)$ with a bimeromorphic morphism from $X$ to $Y$ over $S$. A minimal fibered surface is a relatively minimal fibered surface $(X, S, \pi)$ satisfying the following condition. For any bimeromorphic morphism from $Y$ to $X$ over $S$, all the relatively minimal models of $Y$ are isomorphic to $X$ over $S$. If a relatively minimal model of a fibered surface is minimal, the relatively minimal model is called the minimal model of the fibered surface. We also define algebraic (relatively) minimal fibered surfaces in the same way except for replacing bimeromorphic morphisms by proper birational morphisms. A prime divisor $E$ on a fibered surface $(X, S, \pi)$ is said to be exceptional if there exists a fibered surface $(Y, S, \rho)$ and a bimeromorphic morphism $\beta: X \rightarrow Y$ over $S$ satisfying the following conditions.

(1) The restriction $\left.\beta\right|_{X-E}$ is an isomorphism.

(2) The image $\beta(E)$ is a point on $Y$. 
The normal sheaf of an analytic subset $D$ of a rigid analytic space $X$ is coherent $\mathscr{O}_{D}$-module $\left.\mathscr{O}_{X}(D)\right|_{D}$. Let us denote this $\mathscr{O}_{D}$-module by $\mathscr{N}_{D / X}$.

Theorem 5.1 (Castelnuovo's criterion). A prime divisor $E$ on a fibered surface $(X, S, \pi)$ is exceptional if and only if the following conditions are satisfied.

(1) The image $\pi(E)$ is a point on $S$.

(2) The cohomology group $H^{1}\left(E, \mathscr{O}_{E}\right)$ vanishes.

(3) The equality $\operatorname{deg}_{E} \mathscr{N}_{E / X}=-\operatorname{dim}_{K} C(E)$ holds where we regard the divisor $E$ as a curve over $K$.

In this case, the divisor $E$ is isomorphic to the projective line over $C(E)$.

Proof. For any bimeromorphic morphism $\phi: X \rightarrow Y$ over $S$ between fibered surfaces, the image of exceptional curves of $\phi$ under $\pi$ is a nowhere dense analytic subset of $S$. Therefore, Lemma 2.4, Chow's theorem (Theorem 8.3), and the GAGA theorems (see [18]) enable us to use algebraic results. Thus, the theorem follows from Castelnuovo's criterion for algebraic fibered surfaces (Theorem 9.3.8 in [21], [26, Chapter 6, p.102], Theorem 3.10 in [19]).

Lemma 5.2. An algebraic fibered surface over an affinoid algebra is relatively minimal (resp. minimal) if and only if its analytification is relatively minimal (resp. minimal).

Proof. By Hopf's theorem (Theorem 2.2), we have only to show that the contraction of an exceptional prime divisor on the analytification of an algebraic fibered surface is the analytification of the algebraic contraction. Thus, the lemma follows from Castelnuovo's criterion (Theorem 5.1), the GAGA theorems, and Castelnuovo's criterion for algebraic fibered surfaces.

The following lemma shows that the analytification commutes with base change.

Lemma 5.3. Let $\mathscr{Y}$ be a locally of finite type scheme over an affinoid algebra A. Then, for a K-algebra homomorphism $A \rightarrow B$ of affinoid algebras, there exists a canonical isomorphism

$$
\mathscr{Y}^{\mathrm{an}} \times_{\mathrm{Sp} A} \mathrm{Sp} B \simeq\left(\mathscr{Y} \times{ }_{\mathrm{Spec} A} \operatorname{Spec} B\right)^{\mathrm{an}}
$$

where $\mathscr{Y}^{\mathrm{an}}$ is the analytification of the A-scheme $\mathscr{Y}$ and $\left(\mathscr{Y} \times{ }_{\operatorname{Spec} A} \operatorname{Spec} B\right)^{\mathrm{an}}$ is the analytification of the $B$-scheme $\mathscr{Y} \times{ }_{\operatorname{Spec} A} \operatorname{Spec} B$.

Proof. We can prove this lemma by the same method as in the proof of Satz 1.9 in [18].

Lemma 5.4. The image of any flat morphism of affinoid spaces is a finite union of affinoid subdomains.

Proof. This lemma is shown in [23, 3.4.8] (see also Proposition 3.1.7 1 in [10]).

Lemma 5.5. For any fibered surface $\pi: X \rightarrow S$ and any étale morphism $\alpha: T \rightarrow S$, the base change $\pi \times{ }_{S} T: X \times{ }_{S} T \rightarrow T$ is also a fibered surface. Moreover, if the arithmetic genus of the fibers of $\pi$ is at least one and the morphism $\alpha$ is surjective, the fibered surface $X$ is minimal if and only if the base change $\pi \times{ }_{S} T: X \times{ }_{S} T \rightarrow T$ is minimal.

Proof. Since the morphism $\alpha$ is étale, by (21.D) Theorem 51 in [22], the base space $T$ and the fiber product $X \times{ }_{S} T$ are regular. This implies the first statement.

Let us show the second statement. By Castelnuovo's criterion (Theorem 5.1) and Lemma 5.4, it suffices to show the case when the base spaces $S$ and $T$ are affinoid spaces. Moreover, by Lemma 2.4, we may assume that the projection $\pi$ is projective. By Chow's 
theorem (Theorem 8.3), Lemma 5.3, and Lemma 5.2, the second statement follows from Proposition 9.3.28 in [21].

A morphism $\rho: Y \rightarrow T$ is said to be locally projective if there exists an admissible covering $\left\{U_{i}\right\}_{i \in I}$ of $T$ such that each base change $\rho \times_{T} U_{i}: Y \times_{T} U_{i} \rightarrow U_{i}$ is projective.

Lemma 5.6. For any proper flat surjective morphism $\rho: Y \rightarrow T$ to a regular curve with fibers of dimension at most one, there exist two morphisms $\alpha_{1}: T_{1} \rightarrow T$ and $\alpha_{12}: T_{2} \rightarrow T_{1}$ satisfying the following conditions.

(1) The morphism $\alpha_{1}$ is an admissible affinoid covering.

(2) The morphism $\alpha_{12}$ is étale, quasi-compact, and surjective.

(3) The base change $\rho \times_{T} T_{2}$ is locally projective.

Proof. This lemma is a special case of Theorem 2.1.4 in [8].

Theorem 5.7 (existence of minimal models). Any fibered surface with fibers of arithmetic genus at least one over a quasi-compact regular curve admits a minimal model.

Proof. The last two lemmas and Theorem 9.3.21 in [21] show that the image of the exceptional divisors is a finite number of points. Thus, Castelnuovo's criterion (Theorem 5.1) implies that there exists a relatively minimal model of the fibered surface. Lemma 2.4 and Corollary 9.3.24 in [21] show that the relatively minimal model is a minimal model of the fibered surface.

\section{DEFORMATION OF DiVISORS}

In this section, we study deformation of quasi-compact effective Cartier divisors on paracompact separated rigid analytic spaces.

6.1. Deformation of Divisors on Quasi-Compact Spaces. Let $D$ be an effective Cartier divisor on a quasi-compact separated rigid analytic space $X$. Let $\left\{U_{i}\right\}_{i \in I}$ be a finite admissible affinoid covering of $X$. Put $\mathscr{U}:=\left\{U_{i}\right\}_{i \in I}$. For each $i \in I$, put $D_{i}:=D \cap U_{i}$. We may write $D_{i}=\operatorname{Sp} B_{i}$ and $U_{i}=\operatorname{Sp} A_{i}$. Assume that the kernel of the natural surjective homomorphism $\theta_{i}: A_{i} \rightarrow B_{i}$ is generated by the single non-zero-divisor $h_{i}$ of $A_{i}$. For each $i, j, k \in I$, put $A_{i j}:=A_{i} \widehat{\otimes} A_{j}, U_{i j}:=\operatorname{Sp} A_{i} \cap \operatorname{Sp} A_{j}, U_{i j k}:=\operatorname{Sp} A_{i} \cap \operatorname{Sp} A_{j} \cap \operatorname{Sp} A_{k}, B_{i j}:=B_{i} \widehat{\otimes} B_{j}$, and $D_{i j}:=D \cap U_{i j}=\operatorname{Sp} B_{i j}$. Let $h_{i j}$ be the invertible element $h_{i} / h_{j}$ of $A_{i j}$.

Let $\delta$ be a special real number. For each $i \in I$, put $V_{i \delta}:=U_{i} \times \operatorname{Sp} K\langle T / \delta\rangle$. Put $Y_{\delta}:=$ $X \times \operatorname{Sp} K\langle T / \delta\rangle$, and $\mathscr{V}_{\delta}:=\left\{V_{i \delta}\right\}_{i \in I}$.

All complete $K$-algebra norms on any affinoid algebra is equivalent to each other (Proposition 2 in [5, 6.1.3] and Corollary 3 in [5, 2.1.8]). For each $i, j \in I$, we set complete $K$-algebra norms on affinoid algebras $A_{i}, A_{i j}, B_{i}$, and $B_{i j}$.

For a presheaf $\mathscr{F}$ of abelian groups on $X$, let us denote the $q$-cochain group (resp. $q$ cocycle group) of $\mathscr{U}$ with coefficient in $\mathscr{F}$ by $C^{q}(\mathscr{U}, \mathscr{F})\left(\operatorname{resp} . Z^{q}(\mathscr{U}, \mathscr{F})\right)$.

Theorem 6.1. We use the above notations. Let $\left\{t_{i}\right\}_{i \in I}$ be a section of the normal sheaf $\mathscr{N}_{D / X}$ of $D$. Assume that the cohomology group $H^{1}\left(D, \mathscr{N}_{D / X}\right)$ vanishes. Then there exist a special real number $\delta, s=\left\{s_{i}\right\}_{i \in I} \in C^{0}\left(\mathscr{V}_{\delta}, \mathscr{O}_{Y_{\delta}}\right)$, and $f=\left\{f_{i j}\right\}_{i, j \in I} \in C^{1}\left(\mathscr{V}_{\delta}, \mathscr{O}_{Y_{\delta}}\right)$ satisfying the following conditions:

(1) For all $i \in I$, the first two terms in the expansion of $s_{i}$ with respect to $T$ is equal to $h_{i}+s_{i 1} T$ where $s_{i 1}$ satisfies the equality $\theta_{i}\left(s_{i 1}\right)=t_{i}$.

(2) For all $i, j, k \in I$, the two equalities $s_{i}=f_{i j} s_{j}$ and $f_{i k}=f_{i j} f_{j k}$ hold.

(3) For all $i, j \in I$, the analytic function $f_{i j}$ is invertible. 
We prove the above theorem. For each $i, j \in I$, we write

$$
s_{i}=h_{i}+\sum_{m=1}^{\infty} s_{i m} T^{m}
$$

and

$$
f_{i j}=h_{i j}+\sum_{m=1}^{\infty} f_{i j m} T^{m}
$$

First, note that it suffices to construct $s \in C^{0}\left(\mathscr{V}_{1}, \mathscr{O}_{Y_{1}}\right)$ and $f \in C^{1}\left(\mathscr{V}_{1}, \mathscr{O}_{Y_{1}}\right)$ that satisfy the conditions (1)-(2). Indeed, if we choose a special real number $\delta$ such that the inequality

$$
\sup _{m \geq 1}\left|\frac{f_{i j m} \delta^{m}}{h_{i j}}\right|_{A_{i j}}<1
$$

holds for all $i, j \in I$, then the condition (3) is fulfilled.

We construct a formal solution. For formal power series $F, G \in A[[T]]$ over an affinoid algebra $A$, if $F \equiv G\left(\bmod T^{\mu+1}\right)$, then we write $F \equiv{ }_{\mu} G$. Put $s_{i 0}:=h_{i}, s_{i 1}:=t_{i}$, and $f_{i j 0}:=h_{i j}$. For $\mu \geq 0$, put

$$
s_{i}^{\mu}:=\sum_{m=0}^{\mu} s_{i m} T^{m}
$$

and

$$
f_{i j}^{\mu}:=\sum_{m=0}^{\mu} f_{i j m} T^{m}
$$

We take a set-theoretical section $l_{i}$ of the surjective homomorphism $\theta_{i}: A_{i} \rightarrow B_{i}$.

Claim. There exist formal power series $\left\{s_{i}\right\}_{i \in I}$ and $\left\{f_{i j}\right\}_{i, j \in I}$ such that the equation

$$
s_{i}^{\mu} \equiv{ }_{\mu} f_{i j}^{\mu} s_{j}^{\mu} \text { on } U_{i j}
$$

holds for all $\mu \geq 0$ and for all $i, j \in I$. If Eq. $(i, j, \mu)$ is satisfied for all $i, j \in I$, then the congruence

holds for all $i, j, k \in I$.

$$
f_{i k}^{\mu} \equiv \mu f_{i j}^{\mu} f_{j k}^{\mu} \text { on } U_{i j k}
$$

Proof. We have only to show the first part. Clearly, Eq. $(i, j, 0)$ holds for all $i, j \in I$.

For $\mu>0$, assume that the summations $\left\{s_{i \mu-1}\right\}_{i \in I}$ and $\left\{f_{i j \mu-1}\right\}_{i, j \in I}$ satisfy Eq. $(i, j, \mu-$ 1) for all $i, j \in I$. We define $s_{\mu}$ and $f_{\mu}$ such that Eq. $(i, j, \mu)$ is fulfilled for all $i, j \in I$. Eq. $(i, j, \mu)$ is equivalent to the equation

$$
f_{i j \mu} h_{j}=s_{i \mu}-h_{i j} s_{j \mu}+g_{i j \mu} \text { on } U_{i j}
$$

where $g_{i j \mu} \in A_{i j}$ is defined by the following congruence:

$$
g_{i j \mu} T^{\mu} \equiv \mu s_{i}^{\mu-1}-f_{i j}^{\mu-1} s_{j}^{\mu-1} \text { on } U_{i j} .
$$

The cochain $\left\{\left.g_{i j \mu}\right|_{D_{i j}}\right\}_{i, j \in I}$ satisfies the one-cocycle condition:

$$
g_{i k \mu}=g_{i j \mu}+h_{i j} g_{j k \mu} \text { on } D_{i j k} .
$$

First, let us show that there exists a solution $s_{\mu}$ of the equation:

$$
s_{i \mu}-h_{i j} s_{j \mu}+g_{i j \mu}=0 \text { on } D_{i j} .
$$


If $\mu=1$, then the equality $g_{i j \mu}=0$ holds. For each $i \in I$, put $s_{i \mu}:=l_{i}\left(t_{i}\right)$. Then, since the family $\left\{t_{i}\right\}$ is a section of the normal sheaf $\mathscr{N}_{D / X}$, Eq. (1) is fulfilled. If $\mu>1$, then by the assumption $H^{1}\left(D, \mathscr{N}_{D / X}\right)=0$ there exists $t_{i \mu} \in B_{i}$ such that the equality

$$
t_{i \mu}-h_{i j} t_{j \mu}+g_{i j \mu}=0 \text { on } D_{i j}
$$

holds. For each $i \in I$, put $s_{i \mu}:=l_{i}\left(t_{i \mu}\right)$. Then $s_{\mu}$ satisfies Eq. (1).

Since the analytic function $s_{i \mu}-h_{i j} s_{j \mu}+g_{i j \mu}$ vanishes on $D_{i j}$ and the restriction $\left.h_{j}\right|_{U_{i j}}$ is a defining function of $D_{i j}$, the analytic function $s_{i \mu}-h_{i j} s_{j \mu}+g_{i j \mu}$ is divisible by $h_{j}$. Set

$$
f_{i j \mu}:=\left(s_{i \mu}-h_{i j \mu} s_{j \mu}+g_{i j \mu}\right) / h_{j} \text { on } U_{i j} .
$$

Then the summations $\left\{s_{i \mu-1}\right\}_{i \in I}$ and $\left\{f_{i j \mu-1}\right\}_{i, j \in I}$ are a solution of the system of the equations $(i, j, \mu)_{i, j \in I}$.

Lemma 6.2. Let $h$ be a defining function of an effective Cartier divisor $D$ on an affinoid space $\operatorname{Sp} A$. Then there exists a positive real number $\alpha$ such that if an element $f$ of $A$ vanishes on $D$, then the equality $|f / h|_{A} \leq \alpha|f|_{A}$ holds.

Proof. Since, for all $K$-linear maps between $K$-normed spaces, continuity is equivalent to boundedness (Corollary 3 [5, 2.1.8]), Banach's theorem (Theorem 1 in [6, I, §3]) shows that the isomorphism $A \rightarrow h A$ is homeomorphism. This proves the lemma.

For each $i, j \in I$, applying the above lemma to the affinoid space $\operatorname{Sp} A_{i j}$ and the defining function $\left.h_{j}\right|_{U_{i j}}$ of $\left.D\right|_{U_{i j}}$, we obtain the positive real number $\alpha_{i j}$. Put $\alpha:=\max _{i, j \in I} \alpha_{i j}$.

We introduce $K$-Banach space norms on cochain groups. Put $A^{q}:=C^{q}\left(\left.\mathscr{U}\right|_{D}, \mathscr{O}_{X}\right)$ and $C^{q}:=C^{q}\left(\left.\mathscr{U}\right|_{D}, \mathscr{N}_{D / X}\right)$. Since the family $\left.\mathscr{U}\right|_{D}$ is a finite admissible affinoid covering of $D$, for each $q$, we may regard the $q$-cochain group $A^{q}$ as an affinoid algebra that is the finite direct sum of the affinoid algebras. Then the $q$-cochain group $A^{q}$ is a $K$-Banach algebra, and the $q$-cochain group $C^{q}$ is an $A^{q}$-Banach space. For each $q=0,1$, we define $q$-coboundary operator $\delta^{q}: C^{q} \rightarrow C^{q+1}$ as follows. For $u=\left\{u_{i}\right\}_{i \in I} \in C^{0}$, put

$$
\left(\delta^{0} u\right)_{i j}:=h_{i j} u_{j}-u_{i} \text { on } U_{i j} \text {. }
$$

For $v=\left\{v_{i j}\right\}_{i, j \in I} \in C^{1}$, put

$$
\left(\delta^{1} v\right)_{i j k}:=h_{i j} v_{j k}-v_{i k}+v_{i j} \text { on } U_{i j k} .
$$

By $Z^{1}$ and $B^{1}$ let us denote the $K$-normed subspaces $\operatorname{Ker} \delta^{1}$ and $\operatorname{Im} \delta^{0}$ of $C^{1}$ respectively.

Claim. There exists a positive real number $\beta$ satisfying the following condition. For any $v \in Z^{1}$, there exists a solution $u \in C^{0}$ of the equation $v=\delta^{0} u$ such that the inequality $|u|_{C^{0}}<\beta|v|_{C^{1}}$ holds.

Proof. Since addition, subtraction, multiplication, and restriction are continuous, the coboundary operators are continuous $K$-linear maps. Therefore, the kernel $Z^{1}$ of $\delta^{1}$ is a $K$-Banach space. Put $\eta:=\delta^{0}: C^{0} \rightarrow Z^{1}$. Since the cohomology group $H^{1}\left(D, \mathscr{N}_{D / X}\right)$ vanishes, the equality $Z^{1}=B^{1}$ holds. Then the $K$-linear map $\eta$ between $K$-Banach spaces is surjective and continuous. Thus, Banach's theorem (Theorem 1 in $[6, \mathrm{I}, \S 3]$ ) shows that the $K$-linear map $\eta$ is open. Therefore, we obtain a desired positive real number $\beta$.

We take $\beta$ in the above claim. Put $t_{\mu}:=\left\{t_{i \mu}\right\}_{i \in I} \in C^{0}$ and $g_{\mu}:=\left\{g_{i j \mu}\right\}_{i, j \in I} \in C^{1}$. If $\mu>1$, then we may assume that $\left|t_{\mu}\right|_{\mathrm{Sp} C^{0}}<\beta\left|g_{\mu}\right|_{\mathrm{Sp} C^{1}}$. By Banach's theorem (Theorem 1 in $[6, \mathrm{I}, \S 3])$, we may take a special real number $\gamma$ such that the inequality $\left|t_{i}(t)\right|_{A_{i}}<\gamma|t|_{B_{i}}$ holds for all $i \in I$ and all $t \in B_{i}$. 
By $R$ let us denote a real number field or an affinoid algebra with a norm. For two formal power series $F=\sum_{m=0}^{\infty} a_{m} T^{m} \in R[[T]]$ and $G=\sum_{m=0}^{\infty} b_{m} T^{m} \in R[[T]]$, we write $F \ll G$ if $\left|a_{m}\right| \leq\left|b_{m}\right|$ for all $m \geq 0$. We set a formal power series over $\mathbb{R}$

$$
P(T):=\frac{b}{5 c} \sum_{m=1}^{\infty} \frac{c^{m} T^{m}}{m^{2}}
$$

where $b$ and $c$ is a positive real number. Then the relation

$$
P(T)^{2} \ll \frac{b}{c} P(T) .
$$

holds. We choose four positive real numbers $a, b, c$, and $d$ satisfying the following inequalities:

(1) $\max _{i \in I}\left|s_{i 1}\right|_{A_{i}}<b / 5$;

(2) $\max _{i, j \in I}\left|h_{i j}\right|_{A_{i j}}<d$;

(3) $\alpha(1+(a b / c)+d)<a$;

(4) $\beta \gamma(1+(a b / c)+d)<c$.

Claim. For all $i, j \in I$ and all $\mu \geq 0$, the relations

$$
f_{i j}^{\mu}-h_{i j} \ll a P(T)
$$

and

$(i, \mu)$

$$
s_{i}^{\mu}-h_{i} \ll P(T)
$$

hold.

Proof. Clearly, Rel. $(i, j, 0)$ and Rel. $(i, 1)$ hold for all $i, j \in I$.

Suppose that $\mu>1$. Assume that Rel. $(i, j, \mu-1)$ and Rel. $(i, \mu)$ hold for all $i, j \in I$. Let us show that Rel. $(i, j, \mu)$ and Rel. $(i, \mu+1)$ hold for all $i, j \in I$. Eq. $(i, j, \mu)$ implies that the congruence

$$
f_{i j \mu} T^{\mu} h_{j} \equiv \mu s_{i}^{\mu}-f_{i j}^{\mu-1} s_{j}^{\mu}
$$

holds. The right side of the above congruence is equal to

$$
s_{i}^{\mu}-h_{i}-\left(f_{i j}^{\mu-1}-h_{i j}\right)\left(s_{j}^{\mu}-h_{j}\right)-h_{i j}\left(s_{j}^{\mu}-h_{j}\right)+h_{i}-\left(f_{i j}^{\mu-1}-h_{i j}\right) h_{j} .
$$

Since the last two terms do not contribute to the term containing $T^{v}$ for each $v \geq \mu$, the relation

$$
s_{i}^{\mu}-f_{i j}^{\mu-1} s_{j}^{\mu} \ll(1+(a b / c)+d) P(T)
$$

holds. Thus, the relation

$$
f_{i j \mu} T^{\mu} h_{j} \ll(1+(a b / c)+d) P(T)
$$

holds. By the choice of $\alpha$, the relation

$$
f_{i j \mu} T^{\mu} \ll \alpha(1+(a b / c)+d) P(T)
$$

holds. Therefore, we obtain Rel. $(i, j, \mu)$. By the definition of $g_{i j \mu+1}$, the congruence

$$
g_{i j \mu+1} T^{\mu+1} \equiv{ }_{\mu+1} s_{i}^{\mu}-f_{i j}^{\mu} s_{j}^{\mu}
$$

holds. The right side of the above congruence is equal to

$$
s_{i}^{\mu}-h_{i}-\left(f_{i j}^{\mu}-h_{i j}\right)\left(s_{j}^{\mu}-h_{j}\right)-h_{i j}\left(s_{j}^{\mu}-h_{j}\right)+h_{i}-\left(f_{i j}^{\mu}-h_{i j}\right) h_{j} .
$$


Since the last two terms do not contribute to the term containing $T^{v}$ for each $v \geq \mu$, the relation

$$
s_{i}^{\mu}-f_{i j}^{\mu} s_{j}^{\mu} \ll(1+(a b / c)+d) P(T)
$$

holds. Thus, the relation

$$
g_{i j \mu+1} T^{\mu+1} \ll(1+(a b / c)+d) P(T)
$$

holds. Since the inequality $\left|s_{i \mu+1}\right|_{A_{i}}<\gamma\left|t_{i \mu+1}\right|_{B_{i}}$ holds, by the choice of $\beta$, the relation

$$
s_{i \mu+1} T^{\mu+1} \ll \beta \gamma(1+(a b / c)+d) P(T)
$$

holds. Therefore, we obtain Rel. $(i, \mu+1)$.

Since the power series $P(T)$ converges on a neighborhood of the origin, the formal solutions converge for some special real number $\delta$, i.e., $s \in C^{0}\left(\mathscr{V}_{\delta}, \mathscr{O}_{Y_{\delta}}\right)$ and $f \in C^{1}\left(\mathscr{V}_{\delta}, \mathscr{O}_{Y_{\delta}}\right)$. This proves Theorem 6.1.

Lemma 6.3. Assume that an analytic subset $Y$ of a rigid analytic space $X$ is defined by analytic functions $f_{1}, \ldots, f_{n}$ on $X$. Let $U$ be an admissible open subset of $X$ containing $Y$. Then there exists a special real number $\varepsilon$ such that the affinoid subdomain $X\left(f_{1} / \varepsilon, \ldots, f_{n} / \varepsilon\right)$ is contained in $U$.

Proof. This lemma is shown in Lemma 2.3 in [16]. See also Lemma 1.1.4 in [7].

We say that a Cartier divisor $D$ admits a global defining function if there exist an admissible open subset $P$ of $X$ containing $D$ and a defining function of $D$ on $P$.

Theorem 6.4. Let $D$ be an effective Cartier divisor on a quasi-compact separated rigid analytic space $X$. Assume that the following conditions are satisfied.

(i) The cohomology group $H^{1}\left(D, \mathscr{N}_{D / X}\right)$ vanishes.

(ii) The normal sheaf $\mathscr{N}_{D / X}$ admits a nowhere vanishing section.

Then the divisor $D$ admits a global defining function.

Proof. By assumption, there exist $\delta$, $s$, and $f$ satisfying the conditions in Theorem 6.1. By the conditions (2)-(3) in the same theorem, we obtain the divisor $E_{\delta}$ on $Y_{\delta}$. By Lemma 6.3 , for all sufficiently small special real number $\eta$, the section $s_{i 1}$ is nowhere vanishing on the affinoid subdomain $\operatorname{Sp} A_{i}^{\prime}$ for all $i \in I$ where $A_{i}^{\prime}:=A_{i}\left\langle h_{i} / \eta\right\rangle$. Choose a special real number $\delta$ such that the inequality

$$
\sup _{m \geq 2}\left|\frac{s_{i m} \delta^{m-1}}{s_{i 1}}\right|_{A_{i}^{\prime}}<1
$$

holds for all $i \in I$. Take a special real number $\mu$ that is less than one. For each $i \in I$, put $U_{i}^{\prime}:=\operatorname{Sp} A_{i}^{\prime}\left\langle s_{i 0} / s_{i 1} \delta \mu\right\rangle$. Since the condition (1) in Theorem 6.1 is satisfied, the restriction of the projection $\pi: E_{\delta} \rightarrow X$ to the preimage $\pi^{-1}\left(U_{i}^{\prime}\right)$ is an isomorphism. Put $P:=\bigcup_{i \in I} U_{i}^{\prime}$. Then, by Lemma 6.5 , the union $P$ is an admissible open subset of $X$. Thus, the composite $P \rightarrow B(\delta)$ of the restriction of the inverse $\left.\pi^{-1}\right|_{P}$ and the second projection $Y_{\delta} \rightarrow B(\delta)$ is a global defining function of $D$. 
6.2. Deformation of Quasi-Compact Divisors. For two coverings $\mathscr{U}$ and $\mathscr{V}$ of a rigid analytic space $X$ and a subset $W$ of $X$, let us denote $\{U \cap V \mid U \in \mathscr{U}, V \in \mathscr{V}\}$ and $\{U \cap W \mid$ $U \in \mathscr{U}\}$ by $\mathscr{U} \cap \mathscr{V}$ and $\mathscr{U} \cap W$ respectively.

Lemma 6.5. Let $\mathscr{U}$ be a family of admissible affinoid open subsets of a quasi-separated rigid analytic space $X$. Assume that the rigid analytic space $X$ admits an admissible affnoid covering $\mathscr{V}$ all of whose covering elements intersect at most finite number of elements of $\mathscr{U}$. Then the family $\mathscr{U} \cap \mathscr{V}$ is an admissible covering of an admissible open subset $\cup \mathscr{U}$ of $X$. Therefore, the family $\mathscr{U}$ is an admissible covering of $\bigcup \mathscr{U}$.

Proof. Put $U:=\bigcup \mathscr{U}$. Let $V$ be an element of $\mathscr{V}$. Since the intersection $U \cap V$ is a finite union of an admissible affinoid open subsets of $V$, Corollary 4 in $[5,9.1 .4]$ shows that the intersection $U \cap V$ is an admissible open subset of $V$. Thus, the union $U$ is an admissible open subset of $X$. The same corollary shows that the family $\mathscr{U} \cap V$ is an admissible covering of $U \cap V$. Therefore, since the family $\mathscr{V} \cap U$ is an admissible covering of $U$, the family $\mathscr{U} \cap \mathscr{V}$ is an admissible covering of $U$. This proves the first assertion. Since the admissible covering $\mathscr{U} \cap \mathscr{V}$ is finer than the covering $\mathscr{U}$, the last assertion follows.

Proposition 6.6. Let $D$ be a quasi-compact analytic subset on a paracompact quasiseparated rigid analytic space $X$. Then there exists a finite admissible covering $\left\{U_{i}\right\}_{i \in I} \cup$ $\left\{U_{0}\right\}$ of $X$ satisfying the following three conditions.

(1) For all $i \in I$, the covering element $U_{i}$ is an affinoid open subset of $X$.

(2) The covering element $U_{0}$ is disjoint from $D$.

We take a family $\left\{f_{i j}\right\}_{j \in J_{i}}$ of defining functions of $D$ on each $U_{i}$. Put $U_{i \varepsilon}:=\{x \in U \mid \forall j \in$ $\left.J_{i},\left|f_{i j}(x)\right| \leq \varepsilon\right\}$.

(3) There exists a special real number $\varepsilon$ such that the restriction $U_{i \varepsilon}$ is disjoint from $U_{0}$ for all $i \in I$.

Proof. Take an admissible affinoid covering $\left\{V_{j}\right\}_{j \in J}$ that is locally of finite type. Put $I:=$ $\left\{j \in J \mid U_{j} \cap D \neq \emptyset\right\}$ and $U:=\bigcup_{j \in J-I} U_{i}$. Then, by Lemma 6.5, the family $\left\{U_{i}\right\}_{i \in I} \cup\left\{U_{0}\right\}$ is an admissible covering of $X$ satisfying the conditions (1) and (2). By the maximal modulus principle (Lemma 6 in $[5,9.1 .4]$ ), we obtain a special real number $\varepsilon$ in the condition (3).

The supremum semi-norm on an affinoid algebra $A$ is the $K$-algebra semi-norm $\mid$. $\left.\right|_{\mathrm{Sp} A}: A \rightarrow \mathbb{R}_{\geq 0}$ defined by the equality:

$$
|f|_{\mathrm{Sp} A}:=\sup _{x \in \mathrm{Sp} A}|f(x)|, \quad f \in A .
$$

Corollary 2 in $[5,3.8 .2]$ shows the following lemma.

Lemma 6.7. For an affinoid algebra $A$, the inequality $|f|_{\mathrm{Sp} A} \leq|f|_{A}$ holds for all $f \in A$.

Let $D$ be a quasi-compact effective Cartier divisor on a paracompact separated rigid analytic space $X$. Assume that the divisor $D$ admits a locally principal covering. By Proposition 6.6, we obtain the admissible covering of $\mathscr{U} \cup\left\{U_{0}\right\}$ of $X$ and the special real number $\varepsilon$ such that the family $\mathscr{U}$ is an admissible covering in Theorem 6.1. Put $V_{0 \delta}:=U_{0} \times \operatorname{Sp} K\langle T / \delta\rangle$.

Theorem 6.8. We use the above notations. Assume that the cohomology group $H^{1}\left(D, N_{D / X}\right)$ vanishes. Then we may take a special real number $\delta$ in Theorem 6.1 satisfying the following additional condition.

(4) For all $i \in I$, the analytic function $s_{i}$ dose not vanish on $V_{i \delta} \cap V_{0 \delta}$. 
Proof. There exist $\delta, s$, and $f$ satisfying the conditions in Theorem 6.1. By the above lemma, we may take a special real number $\delta$ such that the inequality

$$
\sup _{m \geq 1}\left|s_{i m} \delta^{m}\right| \operatorname{Sp} A_{i}<\varepsilon
$$

holds for all $i \in I$. This proves the theorem.

The last two theorems show the following theorem.

Theorem 6.9. Let $D$ be a quasi-compact effective Cartier divisor on a paracompact separated rigid analytic space $X$ satisfying the following conditions.

(i) The cohomology group $H^{1}\left(D, \mathscr{N}_{D / X}\right)$ vanishes.

(ii) The normal sheaf $\mathscr{N}_{D / X}$ admits a nowhere vanishing section.

Then the divisor $D$ admits a global defining function.

\section{Blowing-Down of Exceptional CuRves of The First Kind}

In this section, we show that we can blow down exceptional curves of the first kind on regular surfaces.

An exceptional curve of the first kind is a prime divisor $D$ on a regular surface $X$ satisfying the following conditions.

(1) The cohomology group $H^{1}\left(D, \mathscr{O}_{D}\right)$ vanishes.

(2) The equality $\left.\operatorname{deg}_{D} \mathscr{O}_{D}(D)\right|_{D}=-\operatorname{deg}_{K} C(D)$ holds where we regard the divisor $D$ as a curve over $K$.

In this case, the divisor $D$ is isomorphic to the projective line over $C(D)$. When the surface $X$ is proper, by Proposition 8.15 , the last condition is equivalent to that the self-intersection number $D \cdot D$ is equal to the integer $-\operatorname{dim}_{K} C(D)$ (see Appendix). A local calculation shows that the center of the blowing-up is an exceptional curve of the first kind. In this section, we show the converse.

Theorem 7.1 (Castelnuovo's criterion). Let D be an exceptional curve of the first kind on a regular surface $X$. Then we can blow down $X$ along $D$ to a regular surface.

Hopf's theorem (Theorem 2.2) and the above theorem give a necessary and sufficient condition for relative minimality.

Corollary 7.2 (criterion for relative minimality). A proper regular surface is relatively minimal if and only if the surface does not contain any exceptional curves of the first kind.

In the rest of this section, we prove the above theorem. For the next proposition, we start with preparing terminologies and notations. Let $V$ be an affinoid subdomain of an affinoid space $U$. When $V$ is relatively compact in $U$ (over $K$ ), we write $V \Subset U$. Let $\left\{U_{i}\right\}_{i \in I}$ and $\left\{V_{i}\right\}_{i \in I}$ be two finite families of admissible affinoid open subsets of a rigid analytic space $X$. When $V_{i} \Subset U_{i}$ for all $i$, and the family $\left\{V_{i}\right\}_{i \in I}$ covers a subset $S$ of $X$, the family $\left\{U_{i}\right\}_{i \in I}$ is called a relatively big covering of $S$, and the family $\left\{V_{i}\right\}_{i \in I}$ a relatively small covering of $S$ associated to the relatively big covering $\left\{U_{i}\right\}_{i \in I}$.

Let $\left\{V_{i}\right\}_{i \in I}$ be a relatively small covering of a subset $S$ of a rigid analytic space $X$ associated to the relatively big covering $\left\{U_{i}\right\}_{i \in I}$. When $X$ is quasi-separated, for any subset $J$ of $I$, the family $\left\{U_{j}\right\}_{j \in J}$ is an admissible covering of an admissible open subset $\bigcup_{j \in J} U_{j}$ of $X$ by Lemma 6.5. When $X$ is separated, the family $\left\{\bigcup_{i \in I} U_{i}\right\} \cup\left\{X-\bigcup_{i \in I} V_{i}\right\}$ is an admissible covering of $X$ by Lemma 1.1 in [29]. 
Proposition 7.3 (properness criterion). Let $\pi: X \rightarrow Y$ be a morphism of rigid analytic spaces. Assume that the rigid analytic space $X$ is quasi-separated. Suppose that the preimage of a point $p$ on $Y$ under $\pi$ admits a relatively big covering. Then there exists an admissible open subset $V$ of $Y$ containing $p$ such that the restriction $\left.\pi\right|_{\pi^{-1}(V)}: \pi^{-1}(V) \rightarrow V$ is proper.

Proof. Put $D:=\pi^{-1}(p)$. We choose an admissible affinoid open subset $W$ of $Y$ containing $p$ and a family $\left\{g_{i}\right\}_{i \in I}$ of defining functions of $p$ on $W$. For a special real number $\varepsilon$, we put $W_{\varepsilon}:=\left\{y \in Y|\forall i \in I,| g_{i}(x) \mid \leq \varepsilon\right\}$.

We take a relatively small covering $\left\{V_{i}\right\}_{i \in I}$ of $D$ associated to the relatively big covering $\left\{U_{i}\right\}_{i \in I}$. Since the family $\left\{\pi^{*} g_{i}\right\}_{i \in I}$ is a family of defining functions of $D$ on the preimage $\pi^{-1}(W)$, by Lemma 6.3, we obtain a special real number $\varepsilon$ such that the relation $\pi^{-1}\left(W_{\varepsilon}\right) \subset \bigcup_{i \in I} V_{i}$ holds. Put $Z:=\pi^{-1}\left(W_{\varepsilon}\right)$. Since the relation $V_{i} \cap Z \Subset_{W_{\varepsilon}} U_{i} \cap Z$ holds for all $i \in I$, the restriction $\pi$ to $Z$ is proper. Thus, Lemma 6.5 implies that the admissible open subset $W_{\varepsilon}$ is a desired one.

For a special real number $r$, by $B(r)$ let us denote a one-dimensional closed disk with the radius $r$.

Proof of Castelnuovo's criterion. We have only to show that the divisor $D$ contracts to a regular point. We choose two distinct $C(D)$-rational points 0 and $\infty$ on $D$. We take an admissible affinoid open subset $U$ and two analytic functions $f$ and $g$ on $U$ satisfying the following conditions.

(1) The subset $U$ contains 0 .

(2) The analytic function $f$ defines the divisor $D$ on $U$.

(3) The two analytic functions $f$ and $g$ define the point 0 .

(4) The two analytic functions $f$ and $g$ generate the maximal ideal of the local ring $\mathscr{O}_{X, 0}$.

Since the rigid space $X$ is regular and the divisor $D$ is quasi-compact, Lemma 8.9 implies that the divisor $D$ is covered by a finite number of admissible open subsets to whose element the restriction of the divisor $D$ is defined by a single analytic function. We add $U$ to the covering. Since the rigid space $X$ is separated, by Lemma 6.5, the union $Y$ of the covering elements is an admissible open subset of $X$. Take a covering element $V$ and put $W:=U \cap V$. Applying Lemma 6.3 to the intersection $W$ and the restriction of defining functions of 0 on $V$ to $W$, we may assume that the single element $U$ of the covering contains 0 . Then, by the same method, we may assume that the divisor $(g)$ dose not intersect the other covering elements. We define a divisor $E$ on $Y$ by $D+(g)$. Since the normal bundle of the divisor $E$ is trivial, by Theorem 6.4, we obtain a global defining function $\phi$ of $E$ on a quasi-compact admissible open subset of $X$. Repeating the same procedure for the point $\infty$, we obtain two analytic functions $\phi$ and $\psi$ on a quasi-compact admissible open subset $W$ of $X$ satisfying the following conditions.

(1) A divisor $(\phi)-D$ intersects $D$ at the single point 0 .

(2) A divisor $(\psi)-D$ intersects $D$ at the single point $\infty$.

Then the two analytic functions $\phi$ and $\psi$ define the divisor $D$. We may define a morphism $\pi: W \rightarrow B(r) \times B(r)$ for a sufficiently large special real number $r$. By Proposition 7.3 and Lemma 2.4, for a sufficiently small special real number $\varepsilon$, the restriction of $\pi$ to $\pi^{-1}(B(\varepsilon) \times B(\varepsilon))$ is projective. Chow's theorem (Theorem 8.3) shows that the preimage 
of $B(\varepsilon) \times B(\varepsilon)$ under $\pi$ is the analytification of a regular Noetherian scheme of pure dimension two. Therefore, the theorem follows from Castelnuovo's criterion for such schemes [26, Chapter 6, p102].

\section{APPENDIX}

8.1. Coherent Algebras and Finite Rigid Analytic Spaces. In this subsection, we study relationship between coherent $\mathscr{O}_{X}$-algebras on a rigid analytic space $X$ and finite rigid analytic spaces over $X$. By $\operatorname{Coh} A \lg (X)$ let us denote the category of coherent $\mathscr{O}_{X}$-algebras on a rigid analytic space $X$. By $\operatorname{Fin}(X)$ let us denote the category of finite rigid analytic spaces over $X$. We give an equivalence between the two categories that is compatible with base change. Then we prove that we can algebraize finite rigid analytic spaces over a projective rigid analytic space over an affinoid space.

First, recall that for any $K$-homomorphism $A \rightarrow B$ between affinoid algebras and any finite $A$-module $M$, the completion tensor product $M \widehat{\otimes}_{A} B$ is isomorphic to a finite $B$ module $M \otimes_{A} B$ (Proposition 6 in [5, 3.7.3]). Therefore, for an arbitrary morphism $f: X \rightarrow$ $Y$ of rigid analytic spaces and an arbitrary coherent $\mathscr{O}_{Y}$-module $\mathscr{F}$, we may define the pullback $f^{*} \mathscr{F}$ of $\mathscr{F}$ under $f$, which is a coherent $\mathscr{O}_{X}$-module. In particular, we obtain a functor $f_{\text {CohAlg }}^{*}: \operatorname{CohAlg}(Y) \rightarrow \operatorname{CohAlg}(X)$. We also obtain a functor $f_{\text {Fin }}^{*}: \operatorname{Fin}(Y) \rightarrow \operatorname{Fin}(X)$ by the base change of finite rigid analytic spaces over $X$ under $f$.

A local calculation shows the following projection formula.

Proposition 8.1 (projection formula). Let $\pi: X \rightarrow Y$ be a finite morphism of rigid analytic spaces. Then, for any coherent $\mathscr{O}_{X}$-module $\mathscr{F}$ and any coherent $\mathscr{O}_{Y}$-module $\mathscr{G}$, there exists a canonical isomorphism $\pi_{*}\left(\mathscr{F} \otimes \mathscr{O}_{X} \pi^{*} \mathscr{G}\right) \cong \pi_{*} \mathscr{F} \otimes \mathscr{O}_{Y} \mathscr{G}$.

For a finite rigid analytic space $X^{\prime}$ over $X$, the push-forward of the structure sheaf of $X^{\prime}$ is a coherent $\mathscr{O}_{X}$-algebra (Proposition 5 in $[5,9.4 .2]$ ). This correspondence gives a functor $\phi_{X}: \operatorname{Fin}(X) \rightarrow \operatorname{Coh} A \lg (X)$. Since any finite algebra over an affinoid algebra is an affinoid algebra (Proposition 5 in $[5,6.1 .1]$ ), from a coherent $\mathscr{O}_{X}$-algebra we obtain a finite rigid analytic space over $X$ by pasting spaces and morphisms (Proposition 1 in $[5,9.3 .2]$ and Proposition 1 in $[5,9.3 .3])$. This correspondence gives a functor $\psi_{X}$ : $\operatorname{Coh} A \lg (X) \rightarrow$ Fin $(X)$. Then we obtain the following theorem.

Theorem 8.2. We use the above notations. For an arbitrary rigid analytic space $X$, the functors $\phi_{X}$ and $\psi_{X}$ give an equivalence between the two categories $\operatorname{Coh} \operatorname{Alg}(X)$ and $\operatorname{Fin}(X)$. The equivalence commutes with base change in the following sense. For an arbitrary morphism $f: X \rightarrow Y$ of rigid analytic spaces, the diagram

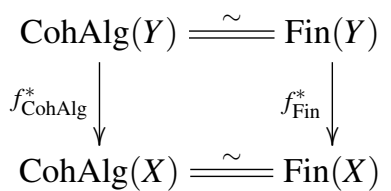

is commutative.

In the rest of this subsection, we prove that we can algebraize finite rigid spaces over a projective rigid analytic space over an affinoid space. We fix an affinoid algebra $A$. Köpf gives the analytification functor from the category of locally of finite type schemes over the affine scheme $\operatorname{Spec} A$ to the category of rigid analytic spaces over the affinoid space $\operatorname{Sp} A$ in $[18, \S 1]$. Let us denote analytification of a locally of finite type scheme $\mathscr{X}$ over $\operatorname{Spec} A$, a morphism $f$ over $\operatorname{Spec} A$ between such schemes, and a coherent $\mathscr{O}_{\mathscr{X}}$-module $\mathscr{F}$ 
by $\mathscr{X}^{\text {an }}, f^{\text {an }}$, and $\mathscr{F}^{\text {an }}$ respectively. When the morphism $f: \mathscr{X} \rightarrow \mathscr{Y}$ is proper, there exists a canonical isomorphism

$$
\left(R^{q} f_{*} \mathscr{F}\right)^{\text {an }} \cong R^{q} f_{*}^{\text {an }} \mathscr{F}^{\text {an }}
$$

for any coherent $\mathscr{O}_{\mathscr{X}}$-module $\mathscr{F}$ and all $q$ (Folgerung 3.13 in [18]). First, note that the following analogue of Chow's theorem follows from the GAGA theorems (Satz 5.1 in [18], Satz 4.11 in [18]).

Theorem 8.3 (Chow's theorem). The analytification functor gives an equivalence between the category of projective schemes over the affine scheme $\operatorname{Spec} A$, where $A$ is an affinoid algebra, and the category of projective rigid analytic spaces over the affinoid space $\mathrm{Sp} A$.

By $\operatorname{Fin}(\mathscr{X})$ let us denote the category of finite schemes over a scheme $\mathscr{X}$.

Theorem 8.4. For any projective scheme $\mathscr{X}$ over an affinoid algebra, the analytification functor gives an equivalence between the category $\operatorname{Fin}(\mathscr{X})$ and the category $\operatorname{Fin}\left(\mathscr{X}^{\mathrm{an}}\right)$. In particular, any finite rigid analytic space over a projective rigid analytic space over an affinoid space is projective.

Proof. Let $F$ be the composite of functors

$$
\operatorname{Fin}(\mathscr{X}) \simeq \operatorname{CohAlg}(\mathscr{X}) \simeq \operatorname{CohAlg}\left(\mathscr{X}^{\text {an }}\right) \simeq \operatorname{Fin}\left(\mathscr{X}^{\text {an }}\right),
$$

where the second functor gives an equivalence by the GAGA theorems. We have only to show that there exists a natural transformation from the functor $F$ to the analytification functor of finite schemes over $\mathscr{X}$. This follows from Theorem 8.2 and Isom. (2) for $q=0$. The last statement follows from Corollaire 6.1.11 in [11].

8.2. Cohomology Groups. We review the theory of cohomology groups and give MayerVietoris sequences of cohomology groups of abelian sheaves.

First, we review some facts on cohomology groups of abelian sheaves on rigid analytic spaces. The category of abelian sheaves on a rigid analytic space is enough injective (see [30]). For a quasi-separated paracohmpact rigid analytic space, Čech cohomology agrees with cohomology (Lemma 2.5.7 in [10] and Remark 2.5.5 in [10]). If a quasi-separated paracompact rigid analytic space is of pure dimension $d$, then the $q$-th cohomology group of any abelian sheaf vanishes for $q>d$ (Corollary 2.5.10 in [10]). The $q$-th cohomology group of any coherent module on an affinoid space vanishes for $q>0$ (Theorem 8,7 in [27], Satz 2.4 in [15]). Therefore, using Leray-Cartan spectral sequence (Corollaire 3.3 [1]) with respect to an admissible affinoid covering, we can calculate cohomology groups of any coherent module on a separated paracompact rigid analytic space. The $q$-th cohomology groups of any $\mathscr{O}_{X}$-coherent module $\mathscr{F}$ on a proper rigid analytic space $X$ is a finite dimensional $K$-vector space (Theorem 3.3 in [13]). Let us denote the dimension of this $K$-vector space by $h^{q}(\mathscr{F})$. In this case, we define the Euler characteristic $\chi(\mathscr{F})$ of $\mathscr{F}$ in the following way:

$$
\chi(\mathscr{F}):=\sum_{q=0}^{\infty}(-1)^{q} \operatorname{dim}_{K} H^{q}(X, \mathscr{F}) .
$$

Since the pull-back of an admissible affinoid covering by a finite morphism is again an admissible affinoid covering (Proposition 1 in $[5,9.4 .4]$ ), we obtain the following proposition.

Proposition 8.5. Let $\pi: X \rightarrow Y$ be a finite morphism between separated paracompact rigid analytic spaces $X$ and $Y$. Then, for a coherent $\mathscr{O}_{X}$-module $\mathscr{F}$, there exists a canonical 
isomorphism

$$
H^{q}(X, \mathscr{F}) \cong H^{q}\left(Y, \pi_{*} \mathscr{F}\right)
$$

for all $q \geq 0$. In particular, if the rigid analytic spaces $X$ and $Y$ are proper, then the equality $\chi(\mathscr{F})=\chi\left(\pi_{*} \mathscr{F}\right)$ holds.

Proposition 8.6 (Mayer-Vietoris sequence). Let $\left\{U_{1}, U_{2}\right\}$ be an admissible covering of an admissible open subset of a rigid analytic space $X$. Then, for any abelian sheaf $\mathscr{F}$, there exists a canonical exact sequence:

$$
\begin{aligned}
0 & \longrightarrow H^{0}\left(U_{1} \cup U_{2},\left.\mathscr{F}\right|_{U_{1} \cup U_{2}}\right) \longrightarrow H^{0}\left(U_{1},\left.\mathscr{F}\right|_{U_{1}}\right) \oplus H^{0}\left(U_{2},\left.\mathscr{F}\right|_{U_{2}}\right) \longrightarrow \\
& \longrightarrow H^{0}\left(U_{1} \cap U_{2},\left.\mathscr{F}\right|_{U_{1} \cap U_{2}}\right) \longrightarrow H^{1}\left(U_{1} \cup U_{2},\left.\mathscr{F}\right|_{U_{1} \cup U_{2}}\right) \longrightarrow \cdots
\end{aligned}
$$

Proof. We use geometric points on rigid analytic spaces (see [30]). Let $\iota_{0}: U_{1} \cup U_{2} \rightarrow X$, $\iota_{1}: U_{1} \rightarrow X, \iota_{2}: U_{2} \rightarrow X$, and $\iota_{3}: U_{1} \cap U_{2} \rightarrow X$ be the inclusion morphisms. Theorem 1 in $[30,4]$ implies that the sheaf sequence

$$
0 \longrightarrow \imath_{0 !}\left(\left.\mathscr{F}\right|_{U_{1} \cup U_{2}}\right) \longrightarrow \iota_{1 !}\left(\left.\mathscr{F}\right|_{U_{1}}\right) \oplus \iota_{2 !}\left(\left.\mathscr{F}\right|_{U_{2}}\right) \longrightarrow \iota_{3 !}\left(\left.\mathscr{F}\right|_{U_{1} \cap U_{2}}\right) \longrightarrow 0 .
$$

is exact. Therefore, we obtain the long exact sequence:

$$
\begin{aligned}
0 \longrightarrow H^{0}\left(X, \iota_{0 !}\left(\left.\mathscr{F}\right|_{U_{1} \cup U_{2}}\right)\right) \longrightarrow H^{0}\left(X, l_{1 !}\left(\left.\mathscr{F}\right|_{U_{1}}\right)\right) \oplus H^{0}\left(X, \iota_{2 !}\left(\left.\mathscr{F}\right|_{U_{2}}\right)\right) \longrightarrow \\
\longrightarrow H^{0}\left(X, \iota_{3 !}\left(\left.\mathscr{F}\right|_{U_{1} \cap U_{2}}\right)\right) \longrightarrow H^{1}\left(X, l_{0 !}\left(\left.\mathscr{F}\right|_{U_{1} \cup U_{2}}\right)\right) \longrightarrow \cdots .
\end{aligned}
$$

For an admissible open subset $U$ of $X$, let $l: U \rightarrow X$ be the inclusion morphism. Then, for any abelian sheaf $\mathscr{G}$ on $U$ and any geometric point $x$ on $X$, we have the isomorphism:

$$
{ }_{l !} \mathscr{G}_{x} \cong \begin{cases}\mathscr{G}_{x}, & x \in U, \\ 0, & \text { otherwise } .\end{cases}
$$

Therefore, when a sheaf sequence $\mathscr{G} \rightarrow \mathscr{I} \bullet$ is the canonical injective resolution, so is the extension $\imath ! \mathscr{G} \rightarrow \imath ! \mathscr{I}^{\bullet}$ of the sheaf sequence. Thus, we obtain the isomorphism

$$
H^{q}(U, \mathscr{G}) \cong H^{q}(X, l ! \mathscr{G})
$$

for all $q$. This proves the proposition.

For a rigid analytic space $X$, by $\mathscr{O}_{X}^{\times}$let us denote the abelian sheaf of units in the sheaf of the ring $\mathscr{O}_{X}$. Then there exists a canonical isomorphism $\operatorname{Pic} X \cong H^{1}\left(X, \mathscr{O}_{X}^{\times}\right)$.

Lemma 8.7. Let $\mathscr{I}$ be a coherent $\mathscr{O}_{X}$-ideal on a paracompact quasi-separated rigid analytic space $X$. Assume that $\mathscr{I}^{2}=0$. Put $X_{0}:=\left(X, \mathscr{O}_{X} / \mathscr{I}\right)$. Let $\pi: X_{0} \rightarrow X$ be the natural closed immersion. Then the sheaf sequence

$$
0 \longrightarrow \mathscr{I} \longrightarrow \mathscr{O}_{X}^{\times} \longrightarrow \pi^{-1} \mathscr{O}_{X_{0}}^{\times} \longrightarrow 0
$$

is exact where, on an admissible affinoid open subset $U$ of $X$, the morphism $\mathscr{I} \rightarrow \mathscr{O}_{X}^{\times}$ is given by $a \mapsto 1+a$ and the the morphism $\mathscr{O}_{X}^{\times} \rightarrow \pi^{-1} \mathscr{O}_{X_{0}}^{\times}$is induced by the natural projection $\mathscr{O}_{X}(U) \rightarrow \mathscr{O}_{X}(U) / \mathscr{I}(U)$. Therefore, we obtain the long exact sequence:

$$
\cdots \longrightarrow H^{1}(X, \mathscr{I}) \longrightarrow \operatorname{Pic} X \longrightarrow \operatorname{Pic} X_{0} \longrightarrow H^{2}(X, \mathscr{I}) \longrightarrow \cdots .
$$

In particular, if the rigid analytic space $X$ is of dimension one, the morphism $\pi^{*}: \operatorname{Pic} X \rightarrow$ Pic $X_{0}$ is surjective.

Proof. Since the sheaf sequence is exact on any affinoid open subset, it is exact at any geometric point on $X$. Therefore, the sheaf sequence is exact. 
8.3. Weil Divisors and Cartier Divisors. We define Weil divisors and Cartier divisors on rigid analytic spaces. Then we prove that these are the same notion on regular rigid analytic spaces. We refer to [4] and [7] for the definition and fundamental results of Weil divisors on normal rigid analytic spaces. We refer to [4] for these of meromorphic functions on rigid analytic spaces.

By $\mathscr{M}_{X}$ let us denote the sheaf of meromorphic functions on a rigid analytic space $X$ (see [4]). A Cartier divisor on $X$ is a global section of the abelian sheaf $\mathscr{M}_{X}^{\times} / \mathscr{O}_{X}^{\times}$. We may represent a Cartier divisor by the family $\left\{\left(U_{i}, f_{i}\right)\right\}_{i \in I}$ where the family $\left\{U_{i}\right\}_{i \in I}$ is an admissible covering of $X$ and $f_{i}$ is a meromorphic function on $U_{i}$. The meromorphic function $f_{i}$ is called a defining function of $D$ on $U_{i}$. A Cartier divisor $D$ is said to be effective if the Cartier divisor $D$ is represented by the family $\left\{\left(U_{i}, f_{i}\right)\right\}_{i \in I}$ where each meromorphic function $f_{i}$ is an analytic function on $U_{i}$. An effective Cartier divisor defines the closed subspace. We sometimes identify a closed subspace with the Cartier divisor. By $\mathscr{O}_{X}(D)$ let us denote the line bundle defined by a Cartier divisor $D$.

When the rigid analytic space $X$ is normal, we may describe Cartier divisors in the following way. A locally principal covering for a Weil divisor $D$ on a normal rigid analytic space $X$ is an admissible covering $\left\{U_{i}\right\}_{i \in I}$ of $X$ such that the restriction of $\left.D\right|_{U_{i}}$ is a principal divisor $\left(f_{i}\right)$ on $U_{i}$. Using this terminology, we may say that a Cartier divisor on $X$ is a Weil divisor on $X$ that admits a locally principal covering. Note that we may assume that a locally principal covering is an admissible affinoid covering if exists.

Lemma 8.8. Any prime Weil divisor $D$ on a regular affinoid space $\operatorname{Sp} A$ admits a finite locally principal Zariski covering.

Proof. Let $I$ be the ideal of $A$ corresponding to $D$. We take a point $x$ on $\operatorname{Sp} A$. Let $\mathfrak{m}$ be the corresponding maximal ideal. Then the localization $A_{\mathfrak{m}}$ is a unique factorization domain since it is a regular local ring. Therefore, we may write $I_{\mathfrak{m}}=f_{x} A_{m}$ where $f_{x} \in A_{m}$. Put $U_{x}:=\operatorname{Sp} A-\operatorname{Supp}\left(D-\left(f_{x}\right)\right)$. By Corollary 7 in [5, 9.1.4], the subset $U_{x}$ is an admissible open subset of $\operatorname{Sp} A$. We take such an admissible open subset for each point $x$ on $\operatorname{Sp} A$. Since $x \in U_{x}$, the family $\left\{U_{x}\right\}_{x \in \operatorname{Sp} A}$ is a Zariski covering of $\operatorname{Sp} A$. We take a finite subcovering $\mathscr{U}$ of this covering. By Corollary 7 in $[5,9.1 .4]$, the covering $\mathscr{U}$ is an admissible covering of $\operatorname{Sp} A$. Since $\left.D\right|_{U_{x}}=\left(f_{x}\right)$, the covering $\mathscr{U}$ is locally principal for $D$.

The above lemma implies the global case.

Theorem 8.9. Any Weil divisor on a regular rigid analytic space $X$ is a Cartier divisor on $X$.

8.4. Proper Rigid Analytic Spaces of Dimension One. In this subsection, we show that any proper rigid analytic space of dimension one is projective.

Since any affinoid algebra is excellent (see [7, 1.1]) and Jacobson (Theorem 3 in [5, 5.2.1]), the singular locus of quasi-compact curve is the union of a finite number of points. A regular divisor on a proper curve $C$ is a formal finite sum of regular points. A prime regular divisor is a regular divisor which is defined by a single regular point. The degree of a regular divisor $\sum_{P} a_{P} P$ is the sum $\sum_{P} a_{P} \operatorname{dim}_{K} K(P)$. Let us denote this integer by $\operatorname{deg}_{C} D$. Since we may regard a regular divisor as a Cartier divisor, a regular divisor $D$ on $C$ defines the line bundle $\mathscr{O}_{C}(D)$ on $C$.

To calculate the dimensions of cohomology groups of line bundles, let us show the following lemma.

Lemma 8.10. Let $P$ be a prime regular divisor on a proper curve $C$. Then, for any line bundle $\mathscr{L}$, the following inequalities and equality hold: 
(1) $0 \leq h^{0}\left(\mathscr{L} \otimes \mathscr{O}_{C}(P)\right)-h^{0}(\mathscr{L}) \leq \operatorname{dim}_{K} K(P)$;

(2) $0 \leq h^{1}(\mathscr{L})-h^{1}\left(\mathscr{L} \otimes \mathscr{O}_{C}(P)\right) \leq \operatorname{dim}_{K} K(P)$;

(3) $\chi\left(\mathscr{L} \otimes \mathscr{O}_{C}(P)\right)=\chi(\mathscr{L})+\operatorname{dim}_{K} K(P)$.

Proof. We define the coherent $\mathscr{O}_{C}$-module $\mathscr{F}$ by the following sheaf exact sequence:

$$
0 \longrightarrow \mathscr{O}_{C}(-P) \longrightarrow \mathscr{O}_{C} \longrightarrow \mathscr{F} \longrightarrow 0 \text {. }
$$

Tensoring the line bundle $\mathscr{L} \otimes \mathscr{O}_{C}(P)$, since the support of cokernel $\mathscr{F}$ is the point $P$, we obtain the sheaf exact sequence:

$$
0 \longrightarrow \mathscr{L} \longrightarrow \mathscr{L} \otimes \mathscr{O}_{C}(P) \longrightarrow \mathscr{F} \longrightarrow 0 \text {. }
$$

Since, by Proposition 8.5 , the equality

$$
h^{i}(C, \mathscr{F})= \begin{cases}\operatorname{dim}_{K} K(P), & i=0, \\ 0, & i \geq 1\end{cases}
$$

holds, the lemma follows from the long exact sequence induced by the above exact sequence.

Lemma 8.11. For any regular divisor $D$ on a proper curve $C$, the equality

$$
\chi\left(\mathscr{O}_{C}(D)\right)=\chi\left(\mathscr{O}_{C}\right)+\operatorname{deg}_{C} D
$$

holds.

Proof. We write $D=\sum_{P} a_{P} P$. By induction on $\left|\sum_{P} a_{P}\right|$, the theorem follows from the equality of the above lemma.

Theorem 8.12. Any proper rigid analytic space of dimension one is projective.

Remark. If the base field $K$ is separably closed, the theorem follows from Theorem 2.1.4 in [8].

Proof. It suffices to show that any proper rigid analytic space of pure dimension one is projective. Moreover, by Lemma 8.7 and Corollary 3.1.6 [9], we have only to show that any proper curve is projective. Let $\bigcup_{i \in I} C_{i}$ be the irreducible decomposition of a proper curve $C$. Choose a regular point $P_{i}$ on each $C_{i}$. By the above two lemmas, there exists a positive integer $n_{i}$ such that the line bundle $\mathscr{O}_{C}\left(n_{i} P_{i}\right)$ admits a section that is not a section of $\mathscr{O}_{C}\left(\left(n_{i}-1\right) P_{i}\right)$. The section gives the meromorphic function $f_{i}$, which is analytic except for the pole at the point $P_{i}$. Let $f$ be the summation of all the meromorphic functions $f_{i}$. Then the restriction of $f$ to each $C_{i}$ is non-constant. The meromorphic function $f$ gives the morphism $\phi: C \rightarrow \mathbb{P}_{K}^{1}$ of rigid analytic spaces.

Proposition 4 in $[5,9.6 .2]$ and the following sentences show that the morphism $\phi$ is proper. Therefore, the proper mapping theorem (Proposition 3 in $[5,9.6 .3]$ ) shows that the image $\phi(C)$ is an analytic subset of $\mathbb{P}_{K}^{1}$. We put the reduced structure on $\phi(C)$. Since the morphism $\phi$ is proper, we have the Stein factorization $\mu \circ \lambda: C \rightarrow S \rightarrow \phi(C)$ (Proposition 5 in $[5,9.6 .3])$. By Lemma 4 in $[5,9.6 .3]$, the morphism $\lambda$ is surjective and for any point $s$ on $S$, the preimage $\lambda^{-1}(s)$ is a connected analytic subset of $C$. If the preimage $\lambda^{-1}(s)$ is not a point, then the meromorphic function $f$ is constant on a irreducible component of $C$. This is absurd. Therefore, the preimage $\lambda^{-1}(s)$ is a point on $C$. Thus, Lemma 4 in $[5,9.6 .4]$ shows that the morphism $\lambda$ is an isomorphism. Since the morphism $\mu$ is finite, Theorem 8.4 implies that the rigid analytic space $S$ is projective. Therefore, the proper curve $C$ is projective. 
8.5. Intersection Theory. In this subsection, we develop the intersection theory of Cartier divisors and line bundles on proper surfaces. Then we prove the Riemann-Roch theorem for proper smooth surfaces.

For two line bundles $\mathscr{L}_{1}$ and $\mathscr{L}_{2}$ on a proper surface $X$, we define the intersection number of $\mathscr{L}_{1}$ and $\mathscr{L}_{2}$ by the integer:

$$
\chi\left(\mathscr{O}_{X}\right)-\chi\left(\mathscr{L}_{1}^{\vee}\right)-\chi\left(\mathscr{L}_{2}^{\vee}\right)+\chi\left(\mathscr{L}_{1}^{\vee} \otimes \mathscr{L}_{2}^{\vee}\right) .
$$

Let us denote this integer by $\mathscr{L}_{1} \cdot \mathscr{L}_{2}$. For Cartier divisors $D_{1}$ and $D_{2}$ on $X$ and a line bundle $\mathscr{L}$ on $X$, we abbreviate $D_{1} \cdot D_{2}, D_{1} \cdot \mathscr{L}$, and $\mathscr{L} \cdot D_{1}$ to $\mathscr{O}_{X}\left(D_{1}\right) \cdot \mathscr{O}_{X}\left(D_{2}\right), \mathscr{O}_{X}\left(D_{1}\right) \cdot \mathscr{L}$, and $\mathscr{L} \cdot \mathscr{O}_{X}\left(D_{1}\right)$ respectively. The following proposition is immediate consequence of the definition.

Proposition 8.13. For any two line bundles $\mathscr{L}_{1}$ and $\mathscr{L}_{2}$ on a proper surface $X$, the following equalities hold:

(1) $\mathscr{L}_{1} \cdot \mathscr{O}_{X}=\mathscr{O}_{X} \cdot \mathscr{L}_{1}=0$;

(2) $\mathscr{L}_{1} \cdot \mathscr{L}_{2}=\mathscr{L}_{2} \cdot \mathscr{L}_{1}$.

Lemma 8.14. For any effective Cartier divisor $D$ and any line bundle $\mathscr{L}$ on a proper surface, the following equality holds:

$$
D \cdot \mathscr{L}=\chi\left(\mathscr{O}_{D}\right)-\chi\left(\mathscr{O}_{D} \otimes \mathscr{L}^{\vee}\right) .
$$

Proof. Let $X$ be the given surface. Since we have the sheaf exact sequence

$$
0 \longrightarrow \mathscr{O}_{X}(-D) \longrightarrow \mathscr{O}_{X} \longrightarrow \mathscr{O}_{D} \longrightarrow 0,
$$

tensoring $\mathscr{L}^{\vee}$, we obtain the sheaf exact sequence:

$$
0 \longrightarrow \mathscr{O}_{X}(-D) \otimes \mathscr{L}^{\vee} \longrightarrow \mathscr{L}^{\vee} \longrightarrow \mathscr{O}_{D} \otimes \mathscr{L}^{\vee} \longrightarrow 0
$$

These sequences give the equalities

$$
\chi\left(\mathscr{O}_{D}\right)=\chi\left(\mathscr{O}_{X}\right)-\chi\left(\mathscr{O}_{X}(-D)\right)
$$

and

$$
\chi\left(\mathscr{O}_{D} \otimes \mathscr{L}^{\vee}\right)=\chi\left(\mathscr{L}^{\vee}\right)-\chi\left(\mathscr{O}_{X}(-D) \otimes \mathscr{L}^{\vee}\right)
$$

Subtracting the second equality from the first equality, we obtain the desired equality.

The fundamental calculation methods are given by the following proposition.

Proposition 8.15. Let $\pi: D^{n} \rightarrow D$ be a normalization of an effective Cartier divisor $D$ on a proper surface. Then, for any two line bundles $\mathscr{L}_{1}$ and $\mathscr{L}_{2}$ on the surface, the following equalities hold:

(1) $D \cdot \mathscr{L}=\operatorname{deg}_{D^{n}} \pi^{*} \mathscr{L}$;

(2) $D \cdot \mathscr{L}_{1} \otimes \mathscr{L}_{2}=D \cdot \mathscr{L}_{1}+D \cdot \mathscr{L}_{2}$

Proof. The first equality follows from the previous lemma, Proposition 7.1.32 in [21], and Proposition 7.3.8 in [21]. Therefore, the second equality holds since the equality

$$
\operatorname{deg}_{D^{n}} \pi^{*}\left(\mathscr{L}_{1} \otimes \mathscr{L}_{2}\right)=\operatorname{deg}_{D^{n}} \pi^{*} \mathscr{L}_{1}+\operatorname{deg}_{D^{n}} \pi^{*} \mathscr{L}_{2}
$$

holds.

A direct calculation of intersection numbers shows the following lemma.

Lemma 8.16. For any three line bundles $\mathscr{L}_{1}, \mathscr{L}_{2}$, and $\mathscr{L}_{3}$ on a proper surface, the following equations are equivalent:

(1) $\mathscr{L}_{1} \cdot \mathscr{L}_{2} \otimes \mathscr{L}_{3}=\mathscr{L}_{1} \cdot \mathscr{L}_{2}+\mathscr{L}_{1} \cdot \mathscr{L}_{3}$ 
(2) $\chi\left(\mathscr{O}_{X}\right)-\chi\left(\mathscr{L}_{1}^{\vee}\right)-\chi\left(\mathscr{L}_{2}^{\vee}\right)-\chi\left(\mathscr{L}_{3}^{\vee}\right)+\chi\left(\mathscr{L}_{1}^{\vee} \otimes \mathscr{L}_{2}^{\vee}\right)+\chi\left(\mathscr{L}_{2}^{\vee} \otimes \mathscr{L}_{3}^{\vee}\right)+\chi\left(\mathscr{L}_{3}^{\vee} \otimes\right.$ $\left.\mathscr{L}_{1}^{\vee}\right)-\chi\left(\mathscr{L}_{1}^{\vee} \otimes \mathscr{L}_{2}^{\vee} \otimes \mathscr{L}_{3}^{\vee}\right)=0$

Lemma 8.17. Let $\mathscr{L}_{1}, \mathscr{L}_{2}$, and $\mathscr{L}_{3}$ be line bundles on a proper surface $X$. Assume that one of these line bundles is isomorphic to $\mathscr{O}_{X}(D)$ where $D$ is an effective Cartier divisor. Then the following equality holds:

$$
\mathscr{L}_{1} \cdot \mathscr{L}_{2} \otimes \mathscr{L}_{3}=\mathscr{L}_{1} \cdot \mathscr{L}_{2}+\mathscr{L}_{1} \cdot \mathscr{L}_{3}
$$

Proof. By Lemma 8.16, it suffices to show that the following equality holds:

$$
D \cdot \mathscr{L}_{1} \otimes \mathscr{L}_{2}=D \cdot \mathscr{L}_{1}+D \cdot \mathscr{L}_{2} .
$$

This equality follows from Proposition 8.15 .

Proposition 8.18. Let $\mathscr{L}_{1}, \mathscr{L}_{2}$ and $\mathscr{L}_{3}$ be three line bundles on a proper surface $X$. Assume that one of these line bundles is isomorphic to $\mathscr{O}_{X}(D)$ where $D$ is a Cartier divisor. Then the following equality holds:

$$
\mathscr{L}_{1} \cdot \mathscr{L}_{2} \otimes \mathscr{L}_{3}=\mathscr{L}_{1} \cdot \mathscr{L}_{2}+\mathscr{L}_{1} \cdot \mathscr{L}_{3} .
$$

Proof. By Lemma 8.16, it suffices to show that the equality

$$
D \cdot \mathscr{L}_{1} \otimes \mathscr{L}_{2}=D \cdot \mathscr{L}_{1}+D \cdot \mathscr{L}_{2} .
$$

holds. We may write $D=D_{1}-D_{2}$ where $D_{1}$ and $D_{2}$ are effective Cartier divisors. Lemma 8.17 shows that, for any line bundle $\mathscr{L}$, the equalities

$$
D \cdot \mathscr{L}=D_{1} \cdot \mathscr{L}+\left(-D_{2}\right) \cdot \mathscr{L}
$$

and

$$
0=D_{2} \cdot \mathscr{L}+\left(-D_{2}\right) \cdot \mathscr{L}
$$

hold. Subtracting the second equality from the first equation, we obtain the equality:

$$
D \cdot \mathscr{L}=D_{1} \cdot \mathscr{L}-D_{2} \cdot \mathscr{L}
$$

Thus, the desired equality follows from Lemma 8.17.

Corollary 8.19. Let $D$ be a Cartier divisor $\sum_{i \in I} a_{i} D_{i}$ on a proper surface. Let $\pi_{i}: D_{i}^{n} \rightarrow D_{i}$ be a normalization of an effective Cartier divisor $D_{i}$. Then, for any line bundle $\mathscr{L}$ on the surface, the following equality holds:

$$
D \cdot \mathscr{L}=\sum_{i \in I} a_{i} \operatorname{deg}_{D_{i}^{n}} \pi_{i}^{*} \mathscr{L} .
$$

Corollary 8.20. For any Cartier divisor $\sum_{i \in I} a_{i} D_{i}$ and any line bundle $\mathscr{L}$ on a proper surface, the following equality holds:

$$
\left(\sum_{i \in I} a_{i} D_{i}\right) \cdot \mathscr{L}=\sum_{i \in I} a_{i} D_{i} \cdot \mathscr{L}
$$

Corollary 8.21. For any two Cartier divisors $\sum_{i \in I} a_{i} D_{i}$ and $\sum_{j \in J} b_{j} D_{j}$ on a proper surface, the following equality holds:

$$
\left(\sum_{i \in I} a_{i} D_{i}\right) \cdot\left(\sum_{j \in J} b_{j} D_{j}\right)=\sum_{i \in I, j \in J} a_{i} b_{j} D_{i} \cdot D_{j} .
$$


We show that local calculations yield the intersection number of two effective Cartier divisors. Let $D_{1}$ and $D_{2}$ be the effective Cartier divisors on a proper surface $X$. Assume that the intersection $D_{1} \cap D_{2}$ consists of a finite number of points. The local intersection number of the divisors $D_{1}$ and $D_{2}$ at a point $p$ on $D_{1} \cap D_{2}$ is the integer $\operatorname{dim}_{K}\left(\mathscr{O}_{X} / \mathscr{O}_{X}\left(-D_{1}\right)+\right.$ $\left.\mathscr{O}_{X}\left(-D_{2}\right)\right)_{p}$. Let us denote this number by $I\left(p, D_{1}, D_{2}\right)$. Since the intersection $D_{1} \cap D_{2}$ is a finite number of points, the sum $\sum_{p \in D_{1} \cap D_{2}} I\left(p, D_{1}, D_{2}\right)$ is finite.

Proposition 8.22. We use the above notations. Then the following equality holds:

$$
D_{1} \cdot D_{2}=\sum_{p \in D_{1} \cap D_{2}} I\left(p, D_{1}, D_{2}\right) \text {. }
$$

Proof. Put

$$
\mathscr{F}:=\mathscr{O}_{X} / \mathscr{O}_{X}\left(-D_{1}\right)+\mathscr{O}_{X}\left(-D_{2}\right)
$$

By definition, the equality

$$
\chi(\mathscr{F})=\sum_{p \in D_{1} \cap D_{2}} I\left(p, D_{1}, D_{2}\right)
$$

holds. Since we have the exact sequence

$$
0 \longrightarrow \mathscr{O}_{X}\left(-D_{1}-D_{2}\right) \longrightarrow \mathscr{O}_{X}\left(-D_{1}\right) \oplus \mathscr{O}_{X}\left(-D_{2}\right) \longrightarrow \mathscr{O}_{X} \longrightarrow \mathscr{F} \longrightarrow 0
$$

we obtain the equality:

$$
\chi(\mathscr{F})=\chi\left(\mathscr{O}_{X}\right)-\chi\left(\mathscr{O}_{X}\left(-D_{1}\right)\right)-\chi\left(\mathscr{O}_{X}\left(-D_{2}\right)\right)+\chi\left(\mathscr{O}_{X}\left(-D_{1}-D_{2}\right)\right) .
$$

Since the right side is equal to $D_{1} \cdot D_{2}$, we obtain the desired equality.

Theorem 8.23 (Riemann-Roch theorem for proper smooth surfaces). For any line bundle $\mathscr{L}$ on a proper smooth surface $X$, the equality

$$
\chi(\mathscr{L})=\frac{\mathscr{L} \cdot \mathscr{K}_{X}^{\vee}-\mathscr{L} \cdot \mathscr{L}^{\vee}}{2}+\chi\left(\mathscr{O}_{X}\right)
$$

holds where $\mathscr{K}_{X}$ is the canonical line bundle $\Omega_{X}^{2}$ on $X$.

Proof. The Serre duality theorem (Theorem in $[29,5.1])$ gives the equality:

$$
\mathscr{L} \cdot \mathscr{K}_{X}^{\vee}=\chi(\mathscr{L})-\chi\left(\mathscr{L}^{\vee}\right)
$$

Adding the above equality to the equality

$$
-\mathscr{L} \cdot \mathscr{L}^{\vee}=\chi(\mathscr{L})+\chi\left(\mathscr{L}^{\vee}\right)-2 \chi\left(\mathscr{O}_{X}\right)
$$

we obtain the desired equality.

Acknowledgments. The author thanks Kenji Ueno for support and suggestions, and Fumiharu Kato for support and helpful comments. This work was supported by Grant-in-Aid for JSPS Fellows (21-1111) from Japan Society for the Promotion of Science.

\section{REFERENCES}

1. M. Artin, A. Grothendieck, and J. L. Verdier, Théorie des topos et cohomologie étale des schémas. Théorie des topos, Lecture Notes in Mathematics, vol. 269, 270, 305, Springer-Verlag, Berlin, 1972, Séminaire de Géométrie Algébrique du Bois-Marie 1963-1964 (SGA 4), Avec la collaboration de N. Bourbaki, P. Deligne et B. Saint-Donat.

2. W. Bartenwerfer, Der erste Riemannsche Hebbarkeitssatz im nichtarchimedischen Fall, J. Reine Angew. Math. 286/287 (1976), 144-163.

3. W. Barth, C. Peters, and A. Van de Ven, Compact complex surfaces, Ergebnisse der Mathematik und ihrer Grenzgebiete (3) [Results in Mathematics and Related Areas (3)], vol. 4, Springer-Verlag, Berlin, 1984. 
4. S. Bosch, Meromorphic functions on proper rigid analytic varieties, Seminar on number theory, 1982-1983 (Talence, 1982/1983), Exp. No. 34, 22 pp., Univ. Bordeaux I, Talence, 1983.

5. S. Bosch, U. Güntzer, and R. Remmert, Non-Archimedean analysis, Grundlehren der Mathematischen Wissenschaften [Fundamental Principles of Mathematical Sciences], vol. 261, Springer-Verlag, Berlin, 1984, A systematic approach to rigid analytic geometry.

6. N. Bourbaki, Éléments de mathématique. Fasc. XV. Livre V: Espaces vectoriels topologiques. Chapitre I: Espaces vectoriels topologiques sur un corps valué. Chapitre II: Ensembles convexes et espaces localement convexes, Actualités Scientifiques et Industrielles, No. 1189. Deuxième édition revue et corrigée, Hermann, Paris, 1966.

7. B. Conrad, Irreducible components of rigid spaces, Ann. Inst. Fourier (Grenoble) 49 (1999), no. 2, $473-541$.

8. Modular curves and rigid-analytic spaces, Pure Appl. Math. Q. 2 (2006), no. 1, part 1, $29-110$.

9. __ Relative ampleness in rigid geometry, Ann. Inst. Fourier (Grenoble) 56 (2006), no. 4, 1049-1126.

10. J. de Jong and M. van der Put, Étale cohomology of rigid analytic spaces, Doc. Math. 1 (1996), no. 1, 1-56 (electronic).

11. A. Grothendieck, Éléments de géométrie algébrique. II. Étude globale élémentaire de quelques classes de morphismes, Inst. Hautes Études Sci. Publ. Math. 8 (1961), 222.

12. H. Hopf, Schlichte Abbildungen und lokale Modifikationen 4-dimensionaler komplexer Mannigfaltigkeiten, Comment. Math. Helv. 29 (1955), 132-156.

13. R. Kiehl, Der Endlichkeitssatz für eigentliche Abbildungen in der nichtarchimedischen Funktionentheorie, Invent. Math. 2 (1967), 191-214.

14. Die de Rham Kohomologie algebraischer Mannigfaltigkeiten über einem bewerteten Körper, Inst. Hautes Études Sci. Publ. Math. 33 (1967), 5-20.

15. $\ldots$, Theorem A und Theorem B in der nichtarchimedischen Funktionentheorie, Invent. Math. 2 (1967), 256-273.

16. M. Kisin, Local constancy in p-adic families of Galois representations, Math. Z. 230 (1999), no. 3, $569-593$.

17. K. Kodaira, On the structure of compact complex analytic surfaces. II, Amer. J. Math. 88 (1966), 682-721.

18. U. Köpf, Über eigentliche Familien algebraischer Varietäten über affinoiden Räumen, Schr. Math. Inst. Univ. Münster (2) 7 (1974), iv+72.

19. S. Lichtenbaum, Curves over discrete valuation rings, Amer. J. Math. 90 (1968), 380-405.

20. J. Lipman, Desingularization of two-dimensional schemes, Ann. Math. (2) 107 (1978), no. 1, 151-207.

21. Q. Liu, Algebraic geometry and arithmetic curves, Oxford Graduate Texts in Mathematics, vol. 6, Oxford University Press, Oxford, 2002, Translated from the French by Reinie Erné, Oxford Science Publications.

22. H. Matsumura, Commutative algebra, second ed., Mathematics Lecture Note Series, vol. 56, Benjamin/Cummings Publishing Co., Inc., Reading, Mass., 1980.

23. F. Mehlmann, Ein Beweis für einen Satz von Raynaud über flache Homomorphismen affinoider Algebren, Schr. Math. Inst. Univ. Münster (2) (1981), no. 19, iv+112.

24. H. Schoutens, Blowing up in rigid analytic geometry, Bull. Belg. Math. Soc. Simon Stevin 2 (1995), no. 4, 399-417.

25. Embedded resolution of singularities in rigid analytic geometry, Ann. Fac. Sci. Toulouse Math. (6) 8 (1999), no. 2, 297-330.

26. I. R. Shafarevich, Lectures on minimal models and birational transformations of two dimensional schemes, Notes by C. P. Ramanujam. Tata Institute of Fundamental Research Lectures on Mathematics and Physics, No. 37, Tata Institute of Fundamental Research, Bombay, 1966.

27. J. Tate, Rigid analytic spaces, Invent. Math. 12 (1971), 257-289.

28. K. Ueno, Compact rigid analytic spaces with special regard to surfaces, Algebraic geometry, Sendai, 1985 , Adv. Stud. Pure Math., vol. 10, North-Holland, Amsterdam, 1987, pp. 765-794.

29. M. van der Put, Serre duality for rigid analytic spaces, Indag. Math. (N.S.) 3 (1992), no. 2, 219-235.

30. M. van der Put and P. Schneider, Points and topologies in rigid geometry, Math. Ann. 302 (1995), no. 1, 81-103.

Department of Mathematics, FACUlty of SCIEnCE, Kyoto University, Kyoto 606-8502, JAPAN

E-mail address: mitsui@math.kyoto-u.ac.jp 